\title{
Exploring the Roles of Diaphanous and Enabled Activity in Shaping the Balance between Filopodia and Lamellipodia
}

\author{
Catarina C.F. Homem* and Mark Peifer*t
}

\begin{abstract}
*Department of Biology and ${ }^{+}$Lineberger Comprehensive Cancer Center, University of North Carolina at Chapel Hill, Chapel Hill, NC 27599-3280
\end{abstract}

Submitted February 19, 2009; Revised August 26, 2009; Accepted October 9, 2009

Monitoring Editor: Marcos Gonzalez-Gaitan

\begin{abstract}
During migration cell protrusions power cell extension and sample the environment. Different cells produce different protrusions, from keratocytes dominated by lamellipodia, to growth cones combining filopodia and lamellipodia, to dendritic spines. One key challenge is to determine how the toolkit of actin regulators are coordinated to generate these diverse protrusive arrays. Here we use Drosophila leading-edge (LE) cells to explore how Diaphanous (Dia)-related formins and Ena/VASP proteins cooperate in this process. We first dissect the Dia regulatory region, revealing novel roles for the GTPase-binding and FH3 domains in cortical localization, filopodial initiation, and lengthening. Second, we provide evidence that activating Dia mobilizes Ena from storage places near the LE to act at the LE. Further, Dia and Ena coIP and can recruit one another to new locations, suggesting cooperation is key to their mechanisms of action. Third, we directly explore the functional relationship between Dia and Ena, varying their levels and activity separately in the same cell type. Surprisingly, although each is sufficient to induce filopodia, together they induce lamellipodia. Our data suggest they work together in a complex and nonadditive way, with the ratio between active Dia and Ena being one factor that modulates the balance between filopodia and lamellipodia.
\end{abstract}

\section{INTRODUCTION}

Cell migration is essential for development and maintenance of animal tissues, and alterations in it are central to many pathological processes. In culture, migration is a dynamic and cyclical process, in which a cell extends a protrusion at its leading edge (LE), followed by rear retraction. Actin regulation is particularly important, because actin polymerization at the LE provides the driving force for protrusion (reviewed in Pollard and Borisy, 2003; Zigmond, 2004a).

Two basic protrusion types occur at the LE of migrating cells (Pollard and Borisy, 2003; Mattila and Lappalainen, 2008): lamellipodia, which are broad protrusions containing branched actin filaments that provide traction force for cell migration, and filopodia, fine processes containing parallel bundled actin filaments that are thought to sense the cell's environment. Different cells produce strikingly different suites of protrusions: for example, fish keratocytes produce large lamellipodia, and neuronal growth cones and Drosophila LE cells produce filopodia separated by lamellipodial veils, whereas neuronal dendrites produce largely filopodium-like spines. One current challenge is to determine the machinery used to generate these diverse sets of protrusions.

One model suggests filopodia originate from Arp2/3-initiated actin filaments in lamellipodia by convergent elongation (Svitkina et al., 2003). In this model a subset of barbed ends in the lamellipodial dendritic network are gathered

This article was published online ahead of print in $M B C$ in Press (http:/ / www.molbiolcell.org/cgi/doi/10.1091/mbc.E09-02-0144) on October 21, 2009.

Address correspondence to: Mark Peifer (peifer@unc.edu). together by proteins that protect these filaments from capping and promote elongation. An alternate model suggests Diaphanous (Dia) nucleated and anticapped filaments are sufficient for filopodia (Steffen et al., 2006; Block et al., 2008). In both models, tip complex proteins are thought to mediate lateral interactions between barbed ends, facilitating bundling and ultimately filopodia extension.

The Arp2/3 complex nucleates branched actin filaments and is thought to be the main effector mediating formation of lamellipodia (Pollard and Borisy, 2003). Formins and Enabled (Ena)/VASP proteins, key regulators of linear actin filament elongation, are thought to regulate filopodia (Peng et al., 2003; Pellegrin and Mellor, 2005; Faix and Rottner, 2006). Ena/VASP proteins are suggested to act as anticapping proteins (Barzik et al., 2005; Applewhite et al., 2007), actin bundlers (Bachmann et al., 1999; Huttelmaier et al., 1999; Laurent et al., 1999; Schirenbeck et al., 2006), and antibranching proteins (Samarin et al., 2003; Breitsprecher et al., 2008), perhaps working by monomer gating (Akin and Mullins, 2008). Through these functions they promote formation of unbranched actin filaments.

Formins nucleate actin filaments, but do so de novo rather than by forming branches (Faix and Grosse, 2006; Goode and Eck, 2007; Pollard, 2007). They also protect growing barbed ends from capping, by competing with capping protein, and by actively promote filament elongation. Actin regulation involves the formin homology domain 1 (FH1) and FH2 domains (see Figure 1A), which mediate nucleation, barbed-end binding, and anticapping (Pruyne et al., 2002; Sagot et al., 2002; Zigmond et al., 2003).

Diaphanous-related formins (DRFs) are autoinhibited by intramolecular interactions. The $\mathrm{N}$-terminal regulatory region includes the GTPase-binding domain (GBD) and the FH3 domain (see Figure 1A). The FH3 domain is not as well 
conserved among formins as the other domains, and its function in DRFs is not as clear. At the N-terminus of the FH3 domain is the Diaphanous inhibitory domain (DID), composed of Arm repeats, followed by a dimerization domain (DD; Rose et al., 2005; see Figure 1A). DRFs are turned off by an intermolecular interaction between the Arm repeats/DID and the C-terminal Diaphanous autoinhibitory domain (Dad). Autoinhibition can be relieved when specific small GTPases bind the GBD, displacing Dad from DID (Ridley, 2006). Disrupting this intramolecular interaction by deleting the Dad or GBD generates constitutively active Dia (Alberts, 2001). In the current model, the major role of the GBD is in intramolecular inhibition and its relief by Rho. The DD mediates dimerization, but the biological function of this is not entirely clear. Here we explore novel roles of the GBD, Arm repeats/DID, and DD domains.

Both Ena/VASP and DRFs have roles in filopodia. Ena/ VASP proteins localize to filopodial tips (Lebrand et al., 2004; Gates et al., 2007). Ena/VASP mutants in Drosophila (Gates et al., 2007) and Caenorhabditis elegans (Sheffield et al., 2007) have fewer filopodia. Dia2 localizes at filopodial tips in mammals and in Dictyostelium, and in Dictyostelium is necessary and sufficient for filopodia extension (Schirenbeck et al., 2005a). Ena/VASP and Dia coimmunoprecipitate (coIP) in both Dictyostelium and mice (Grosse et al., 2003; Schirenbeck et al., 2006), but the biological relevance of this remains to be determined. Here we explore this interaction and its implications for how Dia and Ena regulate each other's activity and function.

Previous cell biological and biochemical studies provided a solid foundation of information about the properties of individual actin regulators and are beginning to reveal their individual functions in vivo. However, many actin regulators have overlapping functions in regulating actin networks and cell protrusions. Thus although the Arp2/3 complex was thought to be the primary actin regulator in lamellipodia, a recent study suggests mDia2 also plays a role in lamellipodia and revealed Abi1 as a possible common regulator of both Arp2/3 and formins (Yang et al., 2007). Likewise, in fibroblasts Ena/VASP proteins localize to lamellipodial LEs and regulate persistence of protrusions and thus speed of migration (Bear et al., 2002; Krause et al., 2004), and in keratocytes Ena/VASP proteins at the LE play a role in shaping the smooth leading-edge lamellipodia characteristic of these cells (Lacayo et al., 2007).

This complexity underscores the need to go beyond a focus on a single actin regulator and frames one key challenge for the field: how are different actin regulators coordinately regulated spatially and temporally to produce the right actin structure, facilitating events like directional migration? One simple subset of this broad question is how a cell differentially regulates actin to produce a lamellipodium or filopodium and how different cells use this same machinery to produce different sets of protrusions.

In this study we explore mechanisms by which Dia and Ena modulate cell protrusions, using Drosophila LE cells during dorsal closure as a model. These cells have a complex mix of lamellipodia and filopodia, allowing us to tune protrusions in either direction and providing a chance to observe cells migrating within the complex natural environment of the living embryo. We carry out three sets of experiments. First, we examine how the N-terminal regulatory regions of Dia contribute to its intracellular localization and biological activity. We next ask how Dia and Ena regulate one another's intracellular localization. Finally, we explore how cells integrate the activity of Dia and Ena to determine the type of protrusion formed. Our data reveal
Table 1. Fly stocks and expression patterns, antibodies, and probes

Source

\begin{tabular}{|c|c|c|}
\hline & & Source \\
\hline \multicolumn{3}{|l|}{ Fly stocks } \\
\hline UAS-GFP-Actin & \multicolumn{2}{|c|}{$\begin{array}{l}\text { P. Martin (University of Bristol, Bristol, } \\
\text { United Kingdom) }\end{array}$} \\
\hline \multirow[t]{2}{*}{ UAS-FH1FH2 } & \multicolumn{2}{|c|}{ P. Rorth (EMBL, Heidelberg, Germany) } \\
\hline & \multicolumn{2}{|r|}{ Expression patterns } \\
\hline Gal4 drivers & \multirow{2}{*}{\multicolumn{2}{|c|}{$\begin{array}{l}\text { Expressed in epidermal Engrailed stripes } \\
\text { and in a few amnioserosal cells }\end{array}$}} \\
\hline EnGal4 & & \\
\hline \multirow[t]{3}{*}{ PrdGal4 } & \multirow{2}{*}{\multicolumn{2}{|c|}{$\begin{array}{l}\text { Expressed in epidermal Paired stripes and in } \\
\text { some amnioserosal cells } \\
\text { Expression starts at Stage } 12\end{array}$}} \\
\hline & & \\
\hline & Dilution & Source $^{\mathrm{a}}$ \\
\hline \multicolumn{3}{|l|}{ Antibody/probes } \\
\hline Anti-DE-CAD2 & $1: 100$ & DSHB \\
\hline Anti-Arm & $1: 200$ & DSHB \\
\hline Anti-Ena & $1: 200$ & DSHB \\
\hline Anti-HA & $1: 5000$ & B. Duronio (UNC-CH, NC) \\
\hline Anti-Dia & $1: 5000$ & S. Wasserman (UCSD, CA) \\
\hline Alexa-phalloidin & $1: 200$ & Molecular Probes (Carlsbad, CA) \\
\hline $\begin{array}{l}\text { Secondary } \\
\text { antibodies: } \\
\text { Alexa } 488,568 \\
\text { and } 647\end{array}$ & $1: 500$ & Molecular Probes \\
\hline
\end{tabular}

All other fly stocks were from the Bloomington Stock Center, and fly stocks and Gal4 drivers are described at FlyBase (www.flybase. bio.indiana.edu/).

${ }^{a}$ DSHB, Developmental Studies Hybridoma Bank.

novel roles for the GBD and FH3 domain of Dia, reveal Dia and Ena form a complex and can recruit one another to new locations, and suggest that Dia and Ena play complex, nonadditive roles in determining the type of cell protrusion formed.

\section{MATERIALS AND METHODS}

\section{Genetics}

Mutations and Balancer chromosomes are described at FlyBase (flybase.bio.indiana.edu). Fly stocks and their sources are listed in Table 1. Wild type was $y w$. Females carrying UAS-transgenes were crossed to males with GAL4 drivers, and their expression patterns are described in Table 1. Transgenes were cloned into a derivative of pUASp (Rorth, 1998), modified by T. Murphy for Gateway cloning with an N-terminal EGFP or HA tag (www.ciwemb. edu/labs/murphy/Gateway\%20vectors.html). The different domains of Dia are described in Figure 1A. These Dia domains were cloned into pUASp, with a short linker as follows: M-HA/EGFP-HRYTSLYKKAGSAAAPFT-dia (Homem and Peifer, 2008). Unless noted flies were grown at $25^{\circ} \mathrm{C}$.

\section{Image Acquisition and Quantitation}

Embryos were fixed in 4\% formaldehyde in PBS 1:1 heptane for $20 \mathrm{~min}$. For GFP visualization EGTA was added to the fix (final concentration of $8 \mathrm{mM}$ ). Embryos were hand-devitellinized, incubated in primary antibodies overnight with agitation at $4^{\circ} \mathrm{C}$, in secondary antibodies (Alexa, Molecular Probes) for $2 \mathrm{~h}$ at room temperature, and then mounted in Aquapolymount (Polysciences, Warrington, PA) and imaged with either a Zeiss 510 Confocal or a Zeiss Pascal Confocal microscope (Thornwood, NY). Antibodies used are listed in Table 1. Adobe Photoshop 7.0 (San Jose, CA) was used to adjust input levels to span the entire output grayscale and to adjust brightness and contrast. For live imaging, embryos were bleach dechorionated and mounted in halocarbon oil (series 700; Halocarbon Products, River Edge, NJ) between a coverslip and a permeable membrane (Petriperm; Sartorius, Edgewood, NJ). Images were captured every $15 \mathrm{~s}$ using a Perkin Elmer Wallac Ultraview Confocal Imaging System (Norwalk, CT). Image J (http://rsb.info.nih.gov/ ij/) was used for quantitation of lamellipodial area and filopodial number and length. Filopodia were defined as any thin protrusion $(<1.25 \mu \mathrm{m})$ extending 


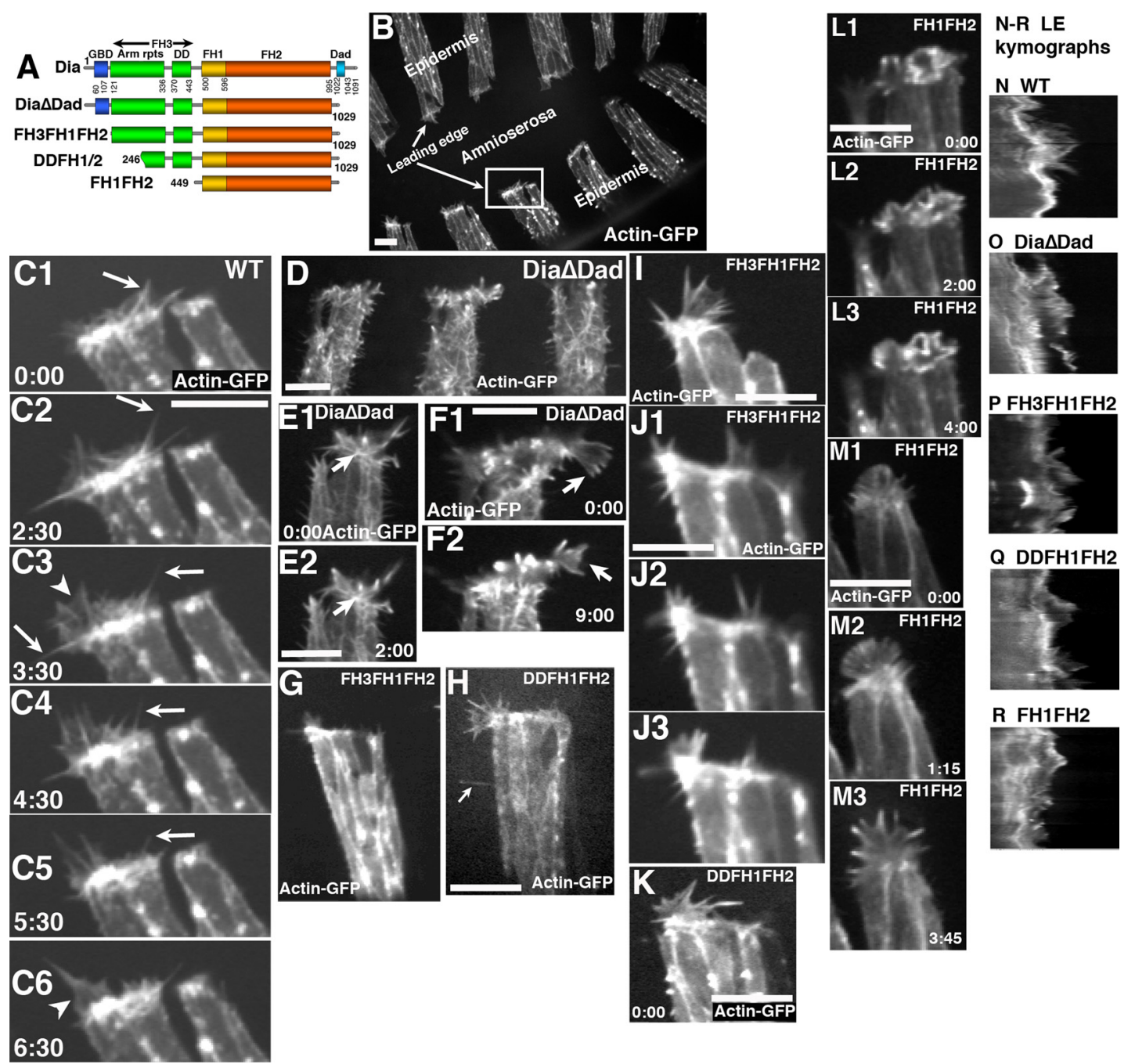

Figure 1. Different Dia mutants have distinct effects on protrusive behavior. (A) Dia constructs. (B-R) Stage 13-14 embryos. Unless noted, all panels in all figures are anterior up and dorsal to the left. Antigens and genotypes indicated. Constructs expressed using en-GAL4. (B) Wild-type living embryo expressing actin-GFP. Inset, region is shown in C1-C6. (C-M) Movie stills. Time is shown in minutes:seconds. (C) Actin-GFP. Arrows, filopodia; arrowheads, lamellipodia. (D-R) Actin-GFP+indicated Dia construct. (D-F) Dia $\Delta$ Dad. (D) Filopodia on more ventral cells. (E) Arrows, leading edge. (F) Arrows, lamellipodia. (G and I) FH3FH1FH2. (H and K) DDFH1FH2. (H) Arrow, single ectopic filopodium. (L and M) FH1FH2. $(\mathrm{N}-\mathrm{R})$ Kymographs of leading edge (LE). The vertical axis is time; 90 frames at $22.5 \mathrm{~min}$ are shown. Scale bars, $10 \mu \mathrm{m}$.

beyond the lamellipodium or LE. Lamellipodia were defined as any projection wider than $1.25 \mu \mathrm{m}$. Lamellipodia area and filopodia number and length were calculated from frames every $2.5 \mathrm{~min}$ within en-GAL4 stripes in three embryos of each genotype (except for Dia $\Delta$ Dad, GFPEna and Dia $\Delta$ Dad, GFPActin, where two embryos were counted) as the LEs moved from 26 to $10.9 \mu \mathrm{m}$ apart. Statistical significance was calculated using the Student's $t$ test (two-sample unequal variance) using Microsoft Excel (Redmond, WA).

\section{Immunoprecipitations}

Dechorionated embryos were homogenized in extraction buffer $(50 \mathrm{mM}$ Tris, $\mathrm{pH} 7.5,1 \% \mathrm{NP}-40,0.5 \mathrm{mM}$ sodium vanadate, $3 \mathrm{mM}$ hydrogen peroxide, Complete EDTA-free protease inhibitor cocktail; Roche, Indianapolis, IN), and centrifuged to remove particulates. Samples were incubated with antibodies $1 \mathrm{~h}$ at $4^{\circ} \mathrm{C}$. Twenty microliters of packed protein A-Sepharose (SigmaAldrich, St. Louis, MO) was added per $100 \mu \mathrm{l}$ sample for an additional $1.5 \mathrm{~h}$ at $4^{\circ} \mathrm{C}$. After washing with extraction buffer, samples were separated by $6 \%$ SDS-PAGE, and immunoblotted.

\section{RESULTS}

We used Drosophila dorsal closure LE cells to explore the function of different domains of the formin Dia in regulating protrusions, and the mechanisms by which Dia and Ena cooperate to determine the type of cell protrusion. During dorsal closure, epidermal cells elongate and amnioserosal cells apically constrict, thus covering embryos in epidermis. LE cells extend protrusions over the amnioserosa that can be visualized with Actin-GFP (Jacinto et al., 2000; Figure 1B, box, and C; Movie 1). These protrusions roughly resemble growth cones, with both lamellipodia (Figure 1C, arrowheads) and filopodia (Figure 1C, arrows; Movie 1; Jacinto et al., 2000; Gates et al., 2007). Filopodial actin bundles appear to form within lamellipodia as microspikes (Figure 1, C1, arrow, 0:00), and a subset protrude beyond the LE to become filopodia (Figure 1, C2, arrow, 2:30; Gates et al., 2007).

Wild-Type Dia Accumulates in the Cytoplasm Where It Is Presumably Inactive

Formins play important roles in protrusive behavior. Dia is the only fly DRF, and we thus explored its role in LE cell protrusive behavior. We began by expressing GFP-tagged wild-type Dia, which retains function (Homem and Peifer, 2008), under control of the GAL4-UAS system, in epidermal 

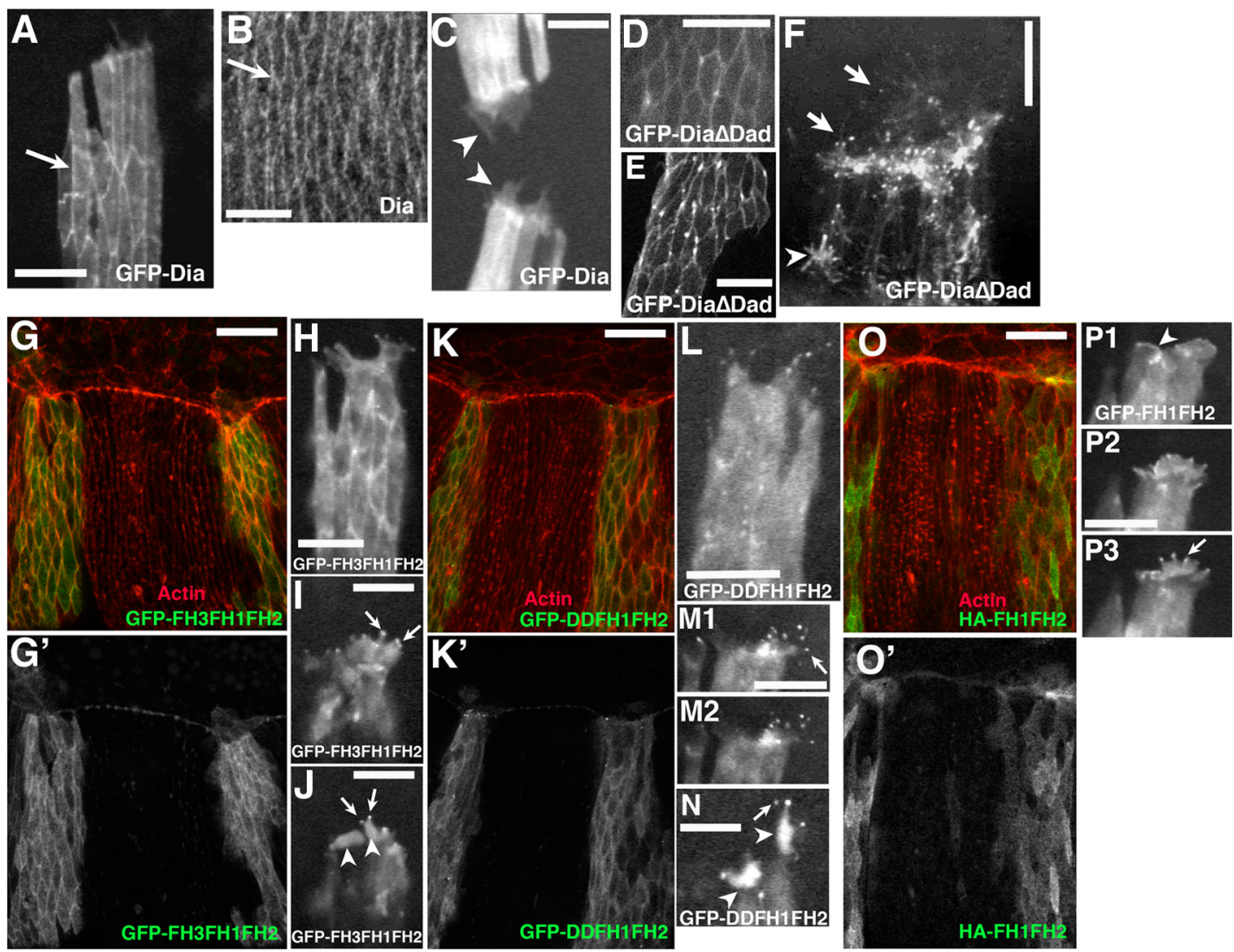

Figure 2. The GBD is important for Dia cortical localization. Embryos, stages 14-15. Antigens and genotypes are indicated. Constructs are expressed with en-GAL4. (A and C) GFP-Dia live. Arrow, cortical enrichment; arrowhead, protrusions. (B) Endogenous Dia at cortex in fixed embryo (arrow). (D-F) GFP-Dia $\Delta$ Dad at cortex in living (D and F) or fixed (E) embryos. Arrows, filopodial tips; arrowhead, enrichment in more ventral filopodia. (G-P) FH3FH1FH2 (G-J), DDFH1FH2 (K-N), and FH1FH2 (O-P) localization in fixed (G, K, and O) and living (H-J, L-N, and P) embryos. (I, J, and M) Arrows, filopodial tips. (J and N) Arrowheads, lamellipodia. (P) Arrowhead, lamellipodial LE. Arrow, "filopodial" tip. Scale bars, $10 \mu \mathrm{m}$

stripes, using either paired-GAL4, expressed in every other segment, or engrailed (en)-GAL4, expressed in posterior cells of each segment. This leads to accumulation at levels roughly paralleling those of endogenous Dia (Homem and Peifer, 2008). Wild-type GFP-Dia accumulates in the cytoplasm and is cortically enriched (Figure 2A; Homem and Peifer, 2008), paralleling endogenous Dia (Figure 2B).

Mammalian and Dictyostelium Dia2 are targeted to filopodial tips (Peng et al., 2003; Pellegrin and Mellor, 2005; Schirenbeck et al., 2005b; Yang et al., 2007) and play roles in forming filopodia. $\mathrm{mDia} 2$ is also enriched in and plays a role in lamellipodia (Yang et al., 2007). We thus hypothesized that GFP-Dia would be enriched in these structures. However, although GFP-Dia went into cell protrusions, it did not accumulate at lamellipodial LEs or filopodial tips (Figure 2C). We also explored whether expressing GFP-Dia affected protrusiveness. We saw no apparent differences in protrusions in these embryos (Supplemental Figure S1A vs. Figure 1C; Movie 2). Together, these data support the hypothesis that the bulk of Dia is in an inactive closed conformation in the cytoplasm and that normal regulatory mechanisms accommodate additional Dia expressed by these GAL4 drivers.

\section{DiasDad Is Recruited to the Cortex and Filopodial Tips and Induces Explosive Formation of Filopodia}

We tested this hypothesis by expressing constitutively activated Dia and examining its localization and effect on pro- trusiveness. Dia is inhibited by intramolecular interactions between the Arm repeats/DID and Dad. We generated Dia $\Delta$ Dad (Figure 1A), which lacks only the Dad and thus should be constitutively active (Alberts, 2001; Rose et al., 2005). Given the known roles of DRFs, we hypothesized Dia $\Delta$ Dad would increase filopodia.

This hypothesis was verified. When we expressed Dia $\Delta$ Dad in stripes of epidermal cells, we saw a dramatic increase in the number of filopodia (Figure 1, D vs. B and C; Movie 3). Most striking, these were no longer confined to LE cells, but instead covered the apical surface of all epidermal cells (Figure 1, D and E). In LE cells filopodia were also triggered all over the cell, rather than being confined to the LE (Figure $1, \mathrm{E}$ and F). At the LE itself, filopodial number was substantially increased (Table 2), and broad lamellipodia terminating in many filopodia were also observed (Figure 1F). Individual filopodia were blunter than in wild type (Figure 1E; Table 2), as seen in mammalian cells (Block et al., 2008). Dia $\Delta$ Dad also more than doubled the lifetime of each filopodium (Table 2).

Deleting the Dad should increase activity by opening up a closed conformation, allowing interaction with new binding partners. This hypothesis predicts that increased activity would correlate with altered intracellular localization. GFPDia $\Delta$ Dad had a strikingly different localization from wildtype Dia, confirming this prediction. The cytoplasmic pool was substantially reduced, and GFP-Dia $\Delta$ Dad was very 
Table 2. Overall protrusive phenotype of different activated Dia mutants

\begin{tabular}{|c|c|c|c|c|c|}
\hline & $\begin{array}{l}\text { Overall phenotype/major } \\
\text { changes from wild type }\end{array}$ & Filopodia number & $\begin{array}{l}\text { Filopodia } \\
\text { length }(\mu \mathrm{m})\end{array}$ & $\begin{array}{l}\text { Lamellipodial } \\
\text { area }\left(\mu \mathrm{m}^{2}\right)\end{array}$ & Filopodial lifetime (s) \\
\hline Wild-type control & $\begin{array}{l}\text { Dynamic mix of filopodia } \\
\text { and lamellipodia }\end{array}$ & $4.7 \pm 0.2$ & $2.6 \pm 0.2$ & $8.5 \pm 0.4$ & $209 \pm 14$ \\
\hline $\operatorname{Dia} \Delta \mathrm{Dad}$ & $\begin{array}{l}\text { Multiple ectopic filopodia no } \\
\text { longer confined to LE }\end{array}$ & $\begin{array}{c}7.2 \pm 0.3 \\
\mathrm{p}<10^{-9} \text { vs. WT }\end{array}$ & $\begin{array}{c}2.32 \pm 0.06 \\
* p=0.13 \text { vs. WT }\end{array}$ & $\begin{array}{c}9.1 \pm 1.1 \\
* \mathrm{p}=0.64 \text { vs. WT }\end{array}$ & $\begin{array}{c}432 \pm 45 \\
\mathrm{p}<10^{-5} \text { vs. WT }\end{array}$ \\
\hline FH3FH1FH2 & $\begin{array}{l}\text { Protrusions very similar to } \\
\text { wild type }\end{array}$ & $\begin{array}{c}4.55 \pm 0.23 \\
* \mathrm{p}=0.70 \text { vs. WT }\end{array}$ & $\begin{array}{c}2.71 \pm 0.07 \\
* \mathrm{p}=0.29 \mathrm{vs} . \mathrm{WT}\end{array}$ & $\begin{array}{c}9.07 \pm 0.61 \\
* \mathrm{p}=0.46 \mathrm{vs} . \mathrm{WT}\end{array}$ & $\begin{aligned} & 262 \pm 15 \\
p= & 0.01 \text { vs. WT }\end{aligned}$ \\
\hline DDFH1FH2 & $\begin{array}{l}\text { Few ectopic filopodia but } \\
\text { filopodia are longer }\end{array}$ & $\begin{array}{c}3.6 \pm 0.3 \\
p<10^{-3} \text { vs. WT }\end{array}$ & $\begin{array}{c}3.1 \pm 0.1 \\
\mathrm{p}=0.007 \text { vs WT }\end{array}$ & $\begin{array}{c}11.4 \pm 0.7 \\
\mathrm{p}<10^{-3} \text { vs. WT }\end{array}$ & $\begin{aligned} & 254 \pm 17 \\
p= & 0.04 \mathrm{vs} . W T\end{aligned}$ \\
\hline \multirow[t]{2}{*}{ FH1FH2 } & \multirow{2}{*}{$\begin{array}{l}\text { Fan-shaped and ruffling } \\
\text { lamellipodia; fewer shorter } \\
\text { filopodia }\end{array}$} & $2.2 \pm 0.2$ & $1.4 \pm 0.1$ & $11.9 \pm 0.5$ & $118 \pm 6$ \\
\hline & & $\mathrm{p}<10^{-16}$ vs. WT & $\mathrm{p}<10^{-10}$ vs. WT & $\mathrm{p}<10^{-6}$ vs. WT & $\mathrm{p}<10^{-7}$ vs. WT \\
\hline
\end{tabular}

Values are means \pm SEM.

* Not statistically significant compared with WT control (i.e., $\mathrm{p}>0.05$ ).

strongly enriched at the cell cortex in both live (Figure 2D) and fixed cells (Figure 2E). As filopodia formed, GFPDia $\Delta$ Dad also accumulated in them and was enriched at their tips, both at the LE and in ectopic filopodia (Figure 2F, arrows and arrowhead, respectively).

\section{All of Our Activated Forms of Dia Elevate Cortical Actin and Disrupt Morphogenesis}

Given these dramatic differences in localization and activity of wild-type Dia and Dia $\Delta$ Dad, we next explored which domains of Dia regulate its localization and activity in epidermal cells. In the current model, the FH1/FH2 domains modulate actin assembly, whereas the GBD, Arm repeats/ DID, and Dad maintain Dia in an inactive state until it is activated by Rho binding (Goode and Eck, 2007). Recent data also implicate the Arm repeats/FH3 in dimerization and interaction with other partners. We thus made several mutant forms of activated Dia (lacking the Dad) that deleted additional domains, to explore their roles in localization and ability to induce filopodia. These were 1) a form of Dia lacking the Dad and the GBD (FH3FH1FH2; Figure 1A); 2) a form of Dia lacking the Dad, GBD, and Arm repeats/DID but retaining the dimerization (DD), FH1 and FH2 domains (DDFH1FH2; Figure 1A; Rose et al., 2005); and 3) the Dia actin modulatory domains with all accessory domains removed (FH1FH2; Figure 1A; originally referred to as DiaCA; Somogyi and Rorth, 2004).

The FH1/FH2 domains are sufficient for actin regulation in vitro (Pollard, 2007) and for elevating f-actin levels in vivo in epidermal cells (Homem and Peifer, 2008). FH3FH1FH2 (Supplemental Figure S2A) and DDFH1FH2 (Supplemental Figure S2B) also are active, elevating cortical actin and altering cell shape from elongated to more rounded when expressed in stripes of epidermal cells, leading to disruption of the normally even LE. Their effects on actin are similar to those of Dia $\Delta$ Dad (Supplemental Figure S2C) and of FH1FH2 (Homem and Peifer, 2008). Interestingly, Dia $\Delta$ Dad had a less severe effect on cell shapes than FH3FH1FH2 and DDFH1FH2. In contrast, a Dia construct similar to FH3FH1FH2 but also carrying the Dad domain did not elevate actin levels, consistent with continued inactivation by DID-Dad interaction in the absence of the GBD (Supplemental Figure S2G).

Dia $\Delta$ Dad and its deletion derivatives are all embryonic lethal when expressed in all epidermal cells using e22c-
GAL4. Analysis of the cuticles secreted by the dead embryos revealed severe defects in the completion of germband retraction, dorsal closure, and head involution (Supplemental Figure S3, A-E). Examination of fixed embryos revealed that ubiquitous expression of Dia $\Delta$ Dad, DDFH1FH2, or FH1FH2 all disrupt normal morphogenesis (Supplemental Figure S3, F-Q), confirming what we observed in the cuticles. Germband retraction is often not completed (Supplemental Figure S3, F, H, J, L, N, and P, arrows), and dorsal closure is quite abnormal, with the LE no longer straight (Supplemental Figure S3, I, K, and Q, arrows) and the zipping together of the two sheets substantially slowed or prevented (Supplemental Figure S3, H and P). These data are consistent with the defects in the actin cytoskeleton described above, and with our previous in depth analysis of the effect of FH1FH2 on morphogenesis (Homem and Peifer, 2008). We have not further characterized these morphogenetic defects, and below focus on effects of these Dia mutants on cell protrusions.

\section{Deleting the GBD Abolishes Ability to Induce Ectopic Filopodia}

Because FH3FH1FH2 and DDFH1FH2 were both active in elevating cortical actin, we hypothesized that both would induce filopodia. We thus expressed them in epidermal stripes. Surprisingly, however, neither induced significant numbers of ectopic filopodia (Figure 1, G and $\mathrm{H})$, unlike Dia $\Delta$ Dad. Further, unlike Dia $\Delta$ Dad (Figure 1D), FH3FH1FH2 and DDFH1FH2 had little effect on protrusions of epidermal cells ventral to the LE (e.g., Figure 1G), inducing only occasional ectopic filopodia (Figure $1 \mathrm{H}$, arrow). Consistent with a reduced ability to induce filopodia, neither FH3FH1FH2 nor DDFH1FH2 induced robust filopodia on amnioserosal cells, whereas Dia $\Delta$ Dad did so (Supplemental Figure S2, D and E vs. F, arrows; data not shown). Instead, LE cells expressing FH3FH1FH2 (Figure 1, I and J; Movie 4) produced protrusions qualitatively similar to those in wild type (Figure 1C). We quantitated both lamellipodial area and filopodial number; both were similar to wild type (Table 2). Further removing the Arm repeats did not have a strong effect; DDFH1FH2 (Figure 1K, Supplemental Figure S1B; Movie 5) also produced protrusions qualitatively like those in wild type, although lamellipodial area and filopodial length were significantly increased (Table 2). Together, these data suggest that the GBD is critical for induction of ectopic filopodia. 


\section{The GBD Plays an Important Role in Cortical Localization}

In parallel, we used these mutant constructs to test the role of the GBD and Arm repeats in Dia localization. Strikingly, both GFP-FH3FH1FH2, and GFP-DDFH1FH2 had localizations substantially different from that of GFP-Dia $\Delta$ Dad. Although GFP-Dia $\Delta$ Dad is largely cortical in either fixed (Figure 2E) or live embryos (Figure 2D), removal of the GBD (FH3FH1FH2) substantially reduced cortical recruitment (Figure 2, G and $\mathrm{H}$ ). Removing the Arm repeats/DID (DDFH1DH2) led to further reduction in cortical recruitment (Figure 2, K-N), and FH1FH2 was also largely cytoplasmic (Figure 2O). These data are consistent with an unexpected role for the GBD and the Arm repeats/DID in cortical recruitment, though it is also possible that differences in Dia activity induce different sorts of actin structures, leading to changes in cortical recruitment. They also further support the hypothesis that cortical recruitment is critical for the ability to induce filopodia.

\section{Is Rho Activation Essential for Cortical Recruitment of Dia?}

One mechanism by which Dia could be recruited to the cortex is by interacting with activated Rho. We thus examined whether activated Rho is essential for Dia cortical recruitment and whether Rho activation enhances it. We first expressed dominant-negative Rho (RhoN19) in segmental stripes using en-GAL4. As previously demonstrated (Bloor and Kiehart, 2002; Magie et al., 2002), this rapidly disrupted adherens junctions (AJs), as assessed by localization of DEcad (Figure 3, $\mathrm{A}^{\prime}$ and $\mathrm{B}^{\prime}$, arrows). Cortical localization of the actin regulator Ena was also reduced (Figure 3, A" and B", arrows). However, this did not abolish Dia cortical localization (Figure 3, $\mathrm{A}^{\prime \prime \prime}$ and $\mathrm{B}^{\prime \prime \prime}$, arrows). Expression of dominant-negative Rho also did not affect the strong cortical localization of Dia $\Delta \mathrm{Dad}$, when they were coexpressed using prd-GAL4 (Figure 3, C and D). Next, we expressed constitutively active Rho (RhoV14) to see if activating Rho elevated levels of cortical Dia. Activation of Rho in segmental stripes leads to deepened segmental grooves, potentially due to accentuated apical constriction, and thus cells expressing RhoCA are in a different focal plane, complicating comparison. There appeared to be some increase in the fraction of Dia at the cortex (Figure 3, E and F, arrow vs. arrowhead), but this might reflect differences in plane of focus in the expressing and nonexpressing cells. Little change in Dia localization was apparent in the amnioserosal cells, where Rho activation was apparent due to increased f-actin levels (Figure 3G, arrow vs. white arrowhead). Together these data suggest Rho is not the only cortical cue, as the GBD can still promote cortical recruitment when Rho is inactivated, but are consistent with the possibility that Rho promotes Dia cortical recruitment.

\section{Active Dia Localizes to Filopodial Tips and Increases Filopodial Lifetime}

Mammalian and Dictyostelium Dia localize to filopodial tips and are thought to promote their elongation. Consistent with this, both GFP-FH3FH1FH2 (Figure 2, I and J, arrows) and GFP-DDFH1FH2 (Figure 2, M and N, arrows) localize to filopodial tips, thus resembling GFP-Dia $\Delta$ Dad. Neither is obviously enriched at lamellipodial edges, though late in dorsal closure both become strongly enriched in the lamellipodial cytoplasm (Figure 2, J and N, arrowheads). This strong localization to filopodial tips also has functional consequences. In addition to promoting the formation of ectopic filopodia, Dia $\Delta$ Dad had a second dramatic effect in the filopodia that are formed. The lifetime of individual filopodia was nearly doubled (Table 2). Although FH3FH1FH2 and DDFH1FH2 did not induce ectopic filopodia, they also significantly increased filopodial lifetime (Table 2). Further, after expression of DDFH1FH2, filopodia were significantly longer than wild type (Table 2; Supplemental Figure S1B, arrows); FH3FH1FH2-expressing embryos also had filopodia with an increased mean length, but this did not reach statistical significance in the sample we measured (Table 2). Thus active Dia increases filopodial lifetime, and deletion of the GBD and Arm-repeats does not abolish this effect.

\section{Removal of the DD Decreases Filopodial Lifetime and Allows Ectopic Activity of FH1FH2}

The FH1 and FH2 domains are the minimum module for actin regulation in vitro (Pollard, 2007) and are sufficient to induce ectopic apical actin, cell shape change, and defects in morphogenesis in vivo in flies (Homem and Peifer, 2008). We thus initially hypothesized they might be sufficient for effects of Dia on filopodia. Unexpectedly, FH1FH2 caused a distinct change in LE cell protrusive behavior. The highly dynamic lamellipodia and filopodia of wild type were replaced by lamellipodia with ruffled edges, which often pointed up into the plane of view instead of projecting over the amnioserosa (Figure 1L; Movie 6), and by fan-shaped lamellipodia with radial actin arrays (Figure 1M2) not observed in wild-type lamellipodia (Figure 1C). Quantitation confirmed that lamellipodia induced by $\mathrm{FH} 1 \mathrm{FH} 2$ were larger than those in wild type (Table 2). In contrast, filopodial number, length and lifetime were all significantly decreased (Table 2). Many of the remaining "filopodia" arose from fan-shaped lamellipodia and did not grow substantially after their appearance (Figures 1M3 and 2P; Movie 7), in contrast to wild-type filopodia or those produced after expression of other activated forms of Dia. Live imaging in cell protrusions revealed that GFP-FH1FH2 is present in the cytoplasm of lamellipodia-like cell protrusions; it is somewhat enriched at LEs of lamellipodial-like structures (Figure $2 \mathrm{P} 1$, arrowhead) and the tips of filopodia that emerge from them (Figure 2P, 1-3, arrow), but filopodial tip recruitment was not as robust as that of GFP-DDFH1FH2 (Figure 2M). These data are consistent with two roles for the DD. First, differences in localization and activity of DDFH1DH2 and FH1FH2 are consistent with the idea that the DD domain plays a role in recruitment to filopodial tips and the ability to stimulate filopodial elongation. Second, because removal of the DD domain switches Dia to promoting ruffles and fanlike lamellipodia, interactions involving the DD domain appear to restrain ectopic activity of $\mathrm{FH} 1 \mathrm{FH} 2$, as these structures were not induced by DDFH1FH2.

Despite the dramatic differences in the nature of the protrusions produced by these different mutants, examination of the LE revealed that in all cases the LE remained quite dynamic. To compare them directly and to complement our measurements of filopodial lifetime, we performed kymography, examining LE lamellipodial dynamics over a period of $22.5 \mathrm{~min}$. The kymographs confirmed the LE was dynamic in genotypes that differed significantly in their qualitative appearance, from wild type (Figure 1N), FH3FH1FH2 (Figure $1 \mathrm{P}$ ), and DDFH1FH2 (Figure 1Q), with a mix of dynamic lamellipodia and filopodia, to Dia $\Delta$ Dad (Figure 1O), with its excess, long-lived ectopic filopodia, to FH1FH2, with its ruffled and fan-like lamellipodia (Figure 1R). 


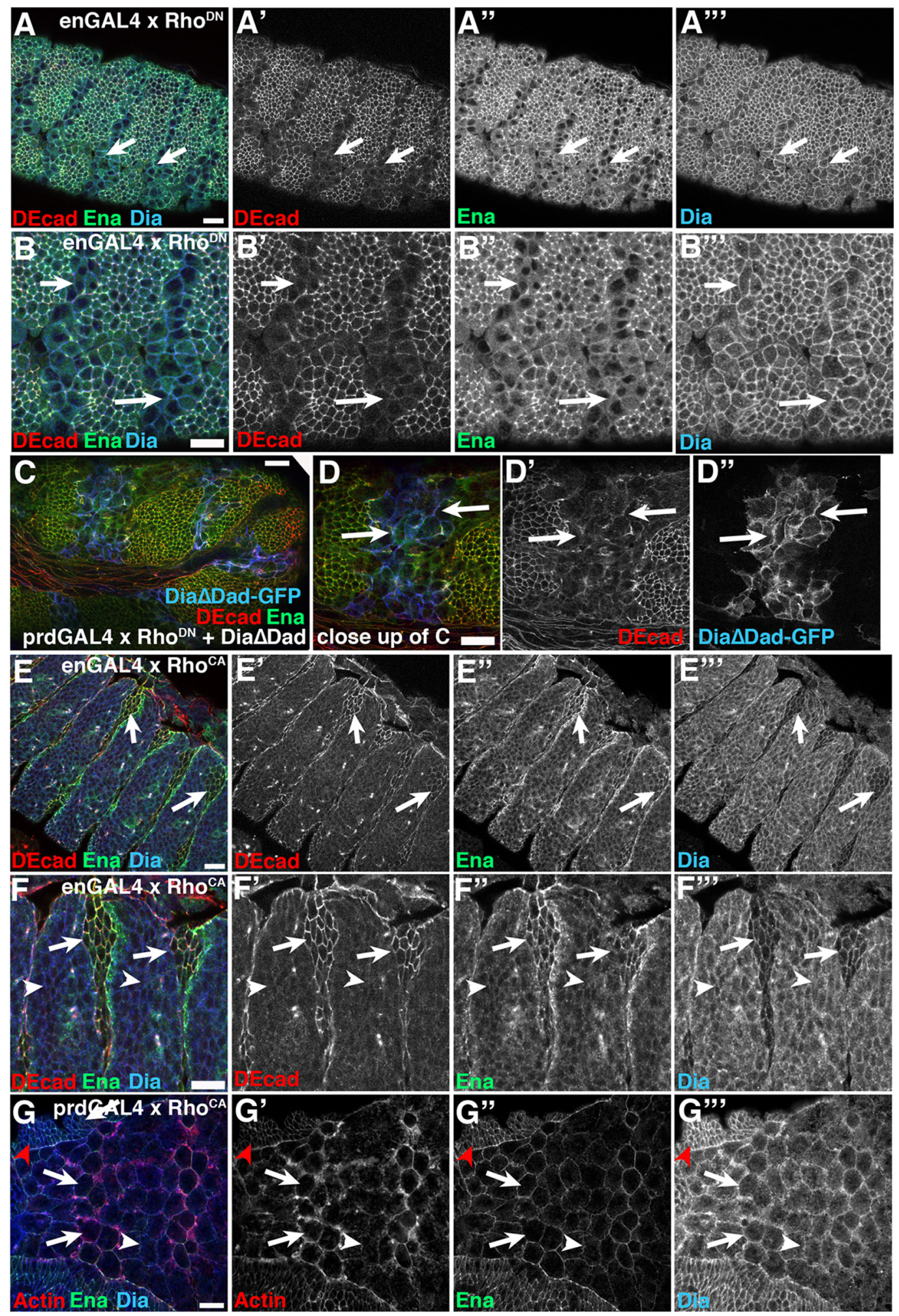

Figure 3. Dominant-negative Rho does not block cortical localization of Dia. Embryos, anterior left and dorsal up, expressing dominantnegative (A-D) or constitutively active (E-G) Rho using en-GAL4 (A, B, E, and F) or prd-GAL4 (C, D, and G). (A and B) Stage 10. Dominant-negative Rho disrupts AJs (arrows), as assessed by DE-cadherin staining, and reduces cortical Ena, but does not reduce cortical Dia. (C and D) Stage 10. Dominant-negative Rho coexpressed with Dia $\Delta$ Dad. Although AJs are disrupted (arrows), Dia $\Delta$ Dad is still highly cortical. (E and F) Stage 13. Constitutively active Rho leads to apical constriction, so expressing cells are found deep in grooves (arrows). Cortical localization of Dia may be increased, though the cell shape change impedes examining exactly the same plane in expressing and nonexpressing cells. For comparison, an apical section through similar nonexpressing cells of another embryo is shown by the red arrowheads in G. (G) Constitutively active Rho elevates cortical actin in expressing (arrow) versus nonexpressing (white arrowheads) amnioserosa cells. Dia is cortical in both. Scale bars, $10 \mu \mathrm{m}$. 

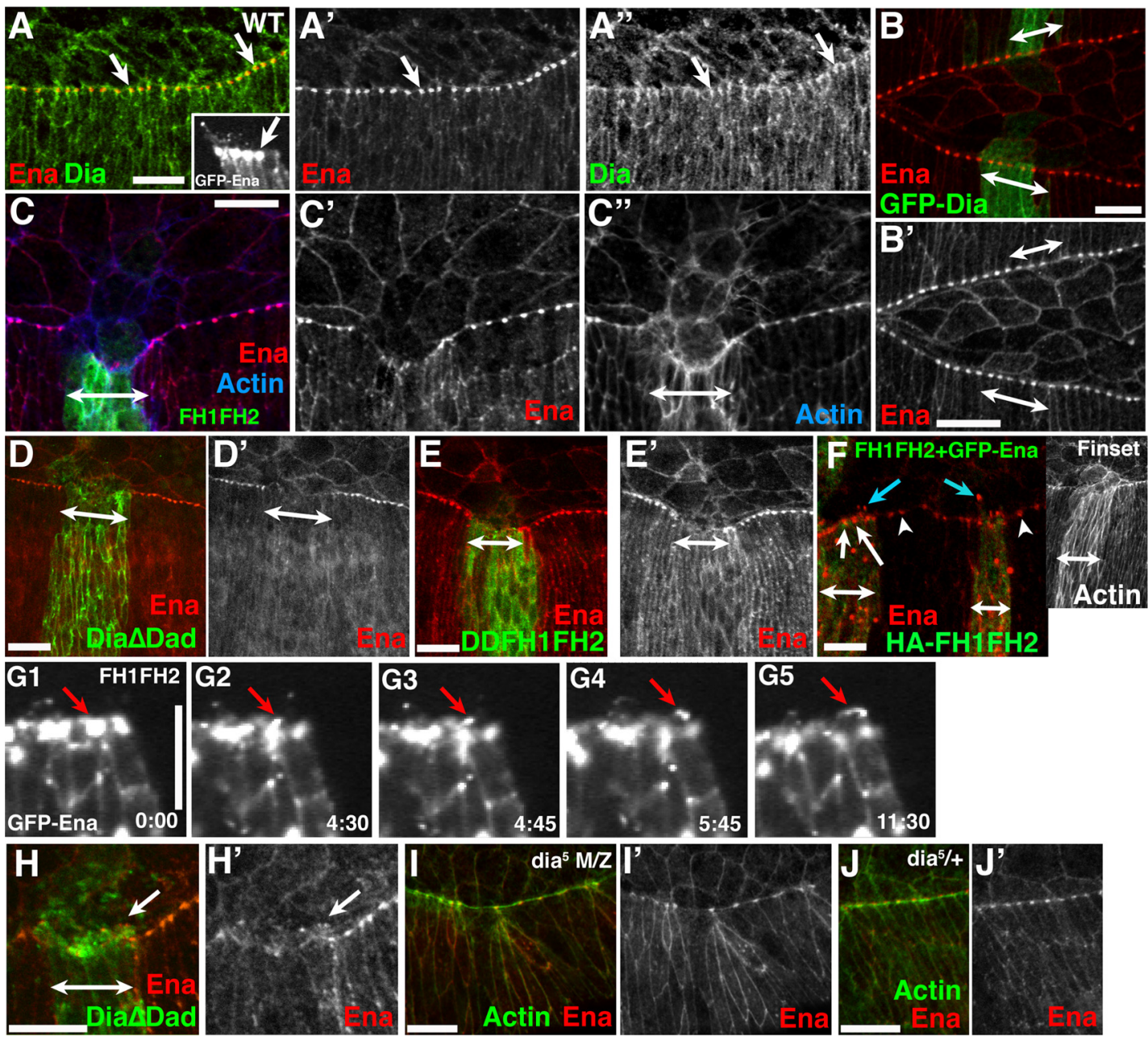

Figure 4. Activated Dia induces Ena relocalization from leading-edge (LE) dots to lamellipodia. Embryos, stages 14-15. Antigens and genotypes are indicated. (A-F and H-J) Fixed embryos. (B-F and H) Double-headed arrows, cells expressing constructs using paired-GAL4 (A) Wild type. Arrow, FH1FH2+GFP-Ena. (F) Inset, actin channel showing elevated actin and disruption of straight leading edge (LE) still occur. (G) Movie stills, embryo expressing GFP-Ena with FH1FH2 using en-Gal4. Time is shown in minutes:seconds. Note relocalization from LE dots (arrow, G1) to edges of lamellipodia (arrows, G2-G5). (H) Ena relocalized to filopodial tips by Dia $\Delta$ Dad. (I and J) Ena remains at LE dots in $\mathrm{dia}^{5} \mathrm{M} / \mathrm{Z}$ mutants (I) and in zygotically rescued siblings (J). Scale bars, $10 \mu \mathrm{m}$.

\section{Activating Dia Induces Ena Relocalization from LE Dots to the LE of Lamellipodia}

Thus different truncated Dia mutants have distinct localizations and induce different protrusions, clarifying roles of $\mathrm{N}$-terminal regulatory domains. One mechanistic difference could be that different Dia deletions utilize different partners. Ena/VASP proteins are also known players in regulating filopodia, and we thus explored the relationship between Dia and Ena in protrusive behavior.

Drosophila has only one Ena/VASP protein. Both mammalian and fly Ena localize at filopodial tips and are required for efficient filopodia formation (Lebrand et al., 2004; Mejillano et al., 2004; Gates et al., 2007). In Drosophila, activation of Ena increases filopodial number and length (Gates et al., 2007), consistent with this role. In mammalian cells, Ena/ VASP proteins also localize to lamellipodial LEs and regulate dynamics of lamellipodial protrusion (Bear et al., 2002).

We previously characterized Ena localization during dorsal closure. Although Ena is present at filopodial tips of LE cells, it accumulates at higher levels at subsets of AJs, both tricellular junctions of all epidermal cells, and especially at LE "dots," where LE cells abut the amnioserosa (Gates et al.,
2007;Figure 4A, arrows). GFP-Ena also localizes to LE dots in live embryos (Figure 4A, inset; Gates et al., 2007). LE dots also have prominent Dia accumulation (Homem and Peifer, 2008; Figure 4A"). Ena's presence at LE dots is puzzling. Ena is not essential for AJ maintenance in Drosophila (Gates et al., 2007, 2009), although it may help link AJs and actin in some mammalian cells (Vasioukhin et al., 2000; Scott et al., 2006). Thus the biological relevance of Ena accumulation at LE dots and Ena's activation state there remained a puzzle.

We hypothesized that LE dots might serve as storage sites for proteins that regulate cell protrusions. To test this hypothesis, we explored how activation of Dia, which dramatically alters protrusive behavior, affects Ena localization. We expressed wild-type Dia or our activated Dia mutants in segmental stripes. Overexpressing GFP-tagged wild-type Dia had no apparent effect on Ena localization (Figure 4B), as it had no effect on protrusive behavior. In contrast, all three activated forms of Dia dramatically altered Ena localization. All induced a striking reduction of Ena at LE dots (Figure 4, C-E; Supplemental Figure S2, A-C). To explore this loss of Ena from LE dots in more detail, we analyzed Ena localization live (in epidermal stripes expressing both GFP-Ena and 
FH1FH2), as FH1FH2 was starting to be expressed. Dia activation triggered GFP-Ena relocalization from LE dots (Figure 4G1, arrow) to newly formed lamellipodial LEs (Figure 4G5, arrow). We could also see relocalized Ena at filopodia in fixed specimens (Figure $4 \mathrm{H}$ ). Interestingly, overexpressing GFP-Ena could partially restore Ena accumulation at LE dots (Figure 4F), although it did not rescue defects in actin organization in LE cells (Figure 4F, inset), suggesting that Ena loss from LE dots is due to recruitment from a limiting pool.

Dia inactivation can destabilize AJs (Carramusa et al., 2007; Homem and Peifer, 2008; Ryu et al., 2009), whereas Dia activation can induce apical constriction and cell shape change (Homem and Peifer, 2008). We thus examined whether loss of Ena at LE dots was a secondary consequence of alterations in AJs. However, although Dia activation promoted pronounced effects in cell shape and actin levels (Supplemental Figure S2; Homem and Peifer, 2008), it did not detectably alter levels or localization of AJ proteins in LE cells. Loss of Ena from LE dots was seen before LE cells begin to elongate (Supplemental Figure S4A), but AJ protein localization was unchanged (Supplemental Figure S4, arrows vs., arrowheads; cells expressing active Dia are in blue; activated Dia induces formation of overly deep segmental grooves, so some cells are below the plane of focus). AJ proteins remained normally localized through LE cell elongation (Supplemental Figure S4B, arrows vs. arrowheads) and later (Supplemental Figure S4C, arrows vs. arrowheads; cells expressing active Dia identified by elevated f-actin). Toward the end of dorsal closure AJ proteins become especially enriched overlapping LE dots (Gorfinkiel and Martinez-Arias, 2007; Supplemental Figure S4, C and D, arrowheads), and this also occurs in cells expressing activated Dia (Supplemental Figure S4, C and D, arrows). Thus Ena recruitment from LE AJs occurs early, before any dramatic AJ rearrangement, and AJs appear normal in LE cells expressing active Dia. Together, these data suggest that Ena is stored at LE dots in an inactive state. We hypothesize that activated Dia recruits Ena from these storage places to the LE, either by creating actin structures that recruit it from LE dots or by a more direct interaction. This further suggests the possibility that Ena recruitment may help shape protrusions induced by Dia activation, and we test this (see below).

\section{Overexpressing Ena Switches Filopodia Produced by Activated Dia to Lamellipodia}

Both Dia and Ena are suggested to act as anticapping proteins, promoting elongation of unbranched actin filaments. Our previous work revealed that Ena localizes to filopodial tips and Ena overexpression/activation alone can increase filopodial number and length (Figure 5, A and B; Gates et al., 2007), consistent with its role in anticapping and filopodial extension. Occasionally we detected Ena along lamellipodial LEs, but this rapidly resolved into filopodia (Figure 5C; Movie 8).

As both Dia and Ena promote filopodia when activated in the wild type, and as activation of Dia recruits Ena from LE dots to the LE, we hypothesized that together they would have additive/synergistic effects on filopodial induction. To our surprise, this was not the case. Instead, coexpressing Ena and activated Dia together induced protrusions strikingly different from those produced by either alone. Dia $\Delta$ Dad induces filopodia in all epidermal cells, even those that normally are not protrusive (Figure 1, D-F). Overexpressing Ena alone increases filopodial length (Gates et al., 2007; Figure 5, A and B; Table 3). However, when we coexpressed Dia $\Delta$ Dad and GFP-Ena, we instead observed lamellipodia, both at the LE (Figure 5, D and E, arrows; Movie 9) and in more ventral epidermal cells (Figure 5, D and E, arrowheads), at the expense of filopodia. Quantitation revealed that these lamellipodia were larger than those seen upon Ena overexpression alone (Figure 5J; Table 3), whereas filopodial length (Figure 5, B vs. F; Table 3) and number were reduced (Figure 5I; Table 3). Consistent with our data above, activating Dia also alters GFP-Ena localization. GFPEna normally localizes to tips of filopodia (Figure 5, A and B, arrow). However, in the presence of activated Dia, GFP-Ena localizes prominently to edges of lamellipodia (Figure 5, D and E, arrows). Thus activating both Dia and Ena pushes protrusiveness from the filopodia each induces alone to lamellipodia.

DDFH1FH2 and FH1FH2 had similar effects. DDFH1FH2 largely converted filopodia induced by Ena overexpression to lamellipodia with GFP-Ena localized along their LEs (Figure 5G; Movie 10); quantitation confirmed increased lamellipodial area and decreased filopodial number (Figure 5, I and J; Table 3). GFP-Ena along the LE sometimes transitioned from relatively evenly distributed to condensing into spots, but these generally did not emerge as filopodia (Figure 5G4). Further, many lamellipodia remained extended $>15$ min (Figure 5G). Coexpression of Ena and FH1FH2 converted the filopodia induced by Ena (Figure 5B) to ruffled lamellipodia, qualitatively similar but significantly larger than those induced by FH1FH2 alone (Figure 5H; Movie 11; Table 3). Together, these data demonstrate that combined activation of Ena and Dia has consequences quite distinct from those caused by activation of either protein alone, consistent with the idea that these two proteins normally cooperate in regulating protrusive behavior, and that they do so in a complex rather than additive manner.

\section{Sequestering Ena Using FP4mito Reduces Lamellipodia But Does Not Prevent Activated Dia from Inducing Filopodia}

To further explore functional interplay between Dia and Ena, we examined consequences of simultaneously activating Dia and reducing Ena activity. We reduced functional Ena at the cell cortex by mislocalization, using Gertler's FP4mito strategy (Bear et al., 2000) as adapted to flies (Gates et al., 2007). FP4mito is GFP fused to four Ena binding sites (FP4 motifs) and a mitochondrial membrane-targeting sequence. FP4mito localizes to the outer mitochondrial membrane, recruits Ena/VASP proteins there, and inactivates Ena/VASP function by removing it from locations where its activity is needed (Bear et al., 2000, 2002; Gates et al., 2007). It sequesters Ena both from the LE (Supplemental Figure S5, A and C, white arrows) and from filopodia (Supplemental Figure S5, B and C, blue arrows) to mitochondria (Supplemental Figure S5, A and C, arrowheads), as assessed by examining localization of either endogenous or GFP-tagged Ena (Gates et al., 2007).

To determine whether full Ena function is essential for the filopodia induced by Dia $\Delta$ Dad, we sequestered Ena using FP4mito. Cells expressing FP4Mito alone generate few filopodia and smooth but smaller lamellipodia (Figure 6, B and I-K; Table 3; Gates et al., 2007). Surprisingly Ena sequestration by FP4Mito did not abolish Dia $\Delta$ Dad's ability to induce elevated numbers of filopodia on LE cells (Figure 6D, arrowheads, and 6I, WT vs. $\Delta$ Dad+FP4; Table 3) or on more ventral epidermal cells (Figure 6D, arrows). This suggests that activated Dia can induce filopodia even when Ena activity is significantly reduced (we verified that Ena still localizes to mitochondria under these conditions; data not shown).

However, although wild-type filopodia emerge from lamellipodia (Figure 6A, arrow), when we sequestered Ena in embryos expressing Dia $\Delta$ Dad, LE filopodia often emerged 

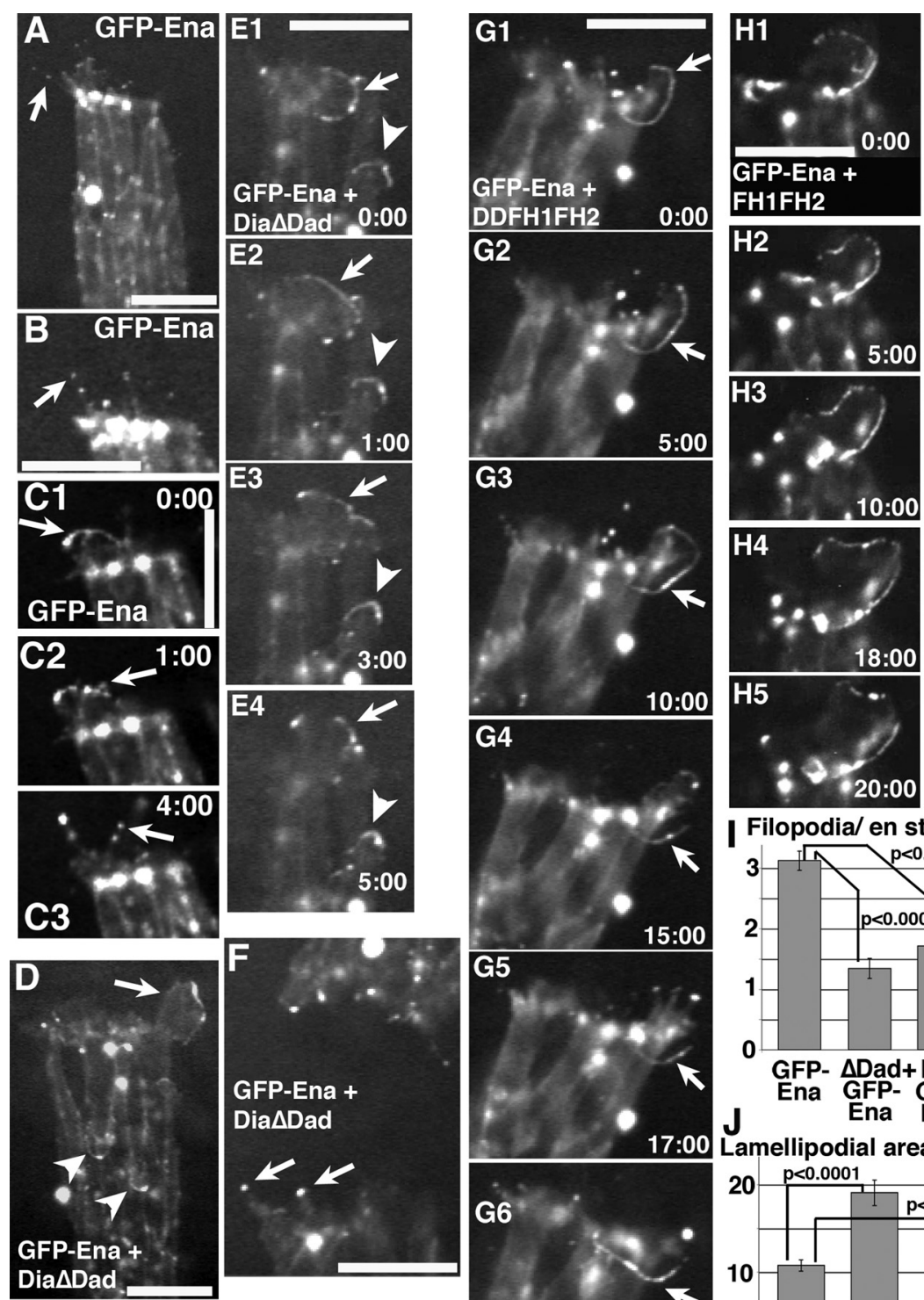

Figure 5. Overexpressing Ena and activating Dia induces lamellipodia rather than filopodia. Movie stills, embryos, stages $14-15$, expressing GFP-Ena alone $(\mathrm{A}-\mathrm{C})$, or with Dia $\Delta$ Dad (D-F), DDFH1FH2 $(G)$, or FH1FH2 $(\mathrm{H})$, using en-Gal4. Time is shown in minutes: seconds. (D and E) Arrows, lamellipodium at leading edge. Arrowheads, lamellipodia in more ventral cells. (F) Arrows, residual filopodia. (G) Arrows, long-lived lamellipodium. Scale bars, $10 \mu \mathrm{m}$. (I and J) Quantitation of number of LE filopodia and lamellipodial area of indicated genotypes. Error bars, SEM.

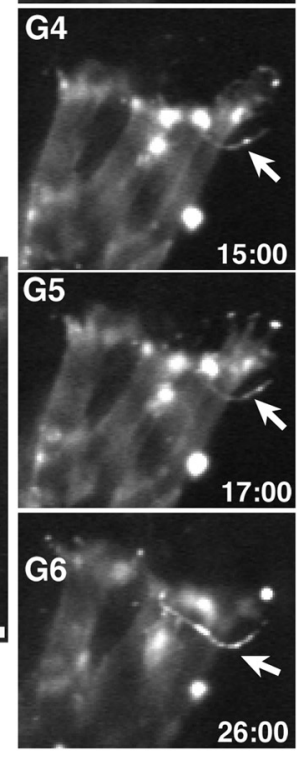

Filopodia/ en stripe

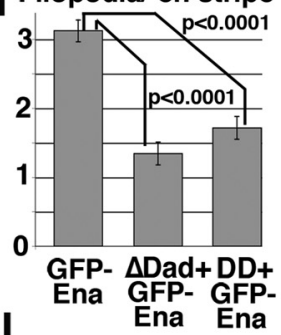

J

Lamellipodial area $\left(\mu \mathrm{m}^{2}\right)$

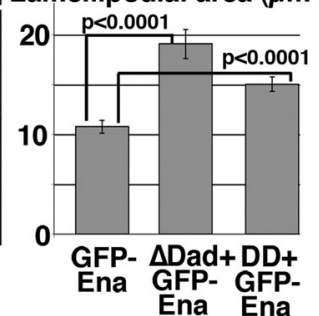

directly from the LE (Figure 6D). Quantitation revealed that the number of lamellipodia is significantly reduced (Figure $6 \mathrm{~K}, \mathrm{WT}$ vs. $\Delta \mathrm{Dad}+\mathrm{FP} 4)$, as is lamellipodial area (Table 3), providing a likely explanation for this. This is consistent with the idea that Ena promotes lamellipodia. Although our previous analysis highlighted the role of Ena in promoting filopodia (Gates et al., 2007), our current analysis revealed that FP4 sequestration of Ena significantly reduces lamellipodial area (Table 3), whereas cells overexpressing Ena have increased lamellipodial area (Table 3). This suggests that in LE cells, Ena promotes lamellipodia. Ena/VASP proteins also regulate the stability of lamellipodia in mammalian fibroblasts (Bear et al., 2002) and fish keratocytes (Lacayo et al., 2007).

When we sequestered Ena in embryos expressing FH1FH2, we also saw an increase in filopodia emerging directly from the LE (Figure 6F). Strikingly, sequestering Ena largely returned filopodia number and length, both of

which are reduced by FH1FH2 alone, to near wild type (Table 3). Further, fewer ruffle-like lamellipodia form (Figure 6, E vs. F; quantitated in Figure 6K, WT vs. $\mathrm{FH} 1 \mathrm{FH} 2+\mathrm{FP} 4)$ and those that form are nearer wild type in size (Table 3; Figure 6, G and H). Thus reducing Ena in the presence of active Dia reduces lamellipodia without eliminating filopodia, suggesting that activated Dia can induce filopodia even when Ena activity is substantially reduced. Further, these data suggest that the abnormal lamellipodia induced by FH1FH2 require Ena, and this may channel actin away from forming filopodia.

\section{Reducing Endogenous Dia Also Biases Ena-overexpressing} Cells toward Lamellipodia

To further explore how Dia and Ena help regulate the type of protrusion formed, we used loss-of-function approaches to reduce each protein. We first reduced endog- 
Table 3. The effect of the Dia/Ena ratio on protrusive behavior

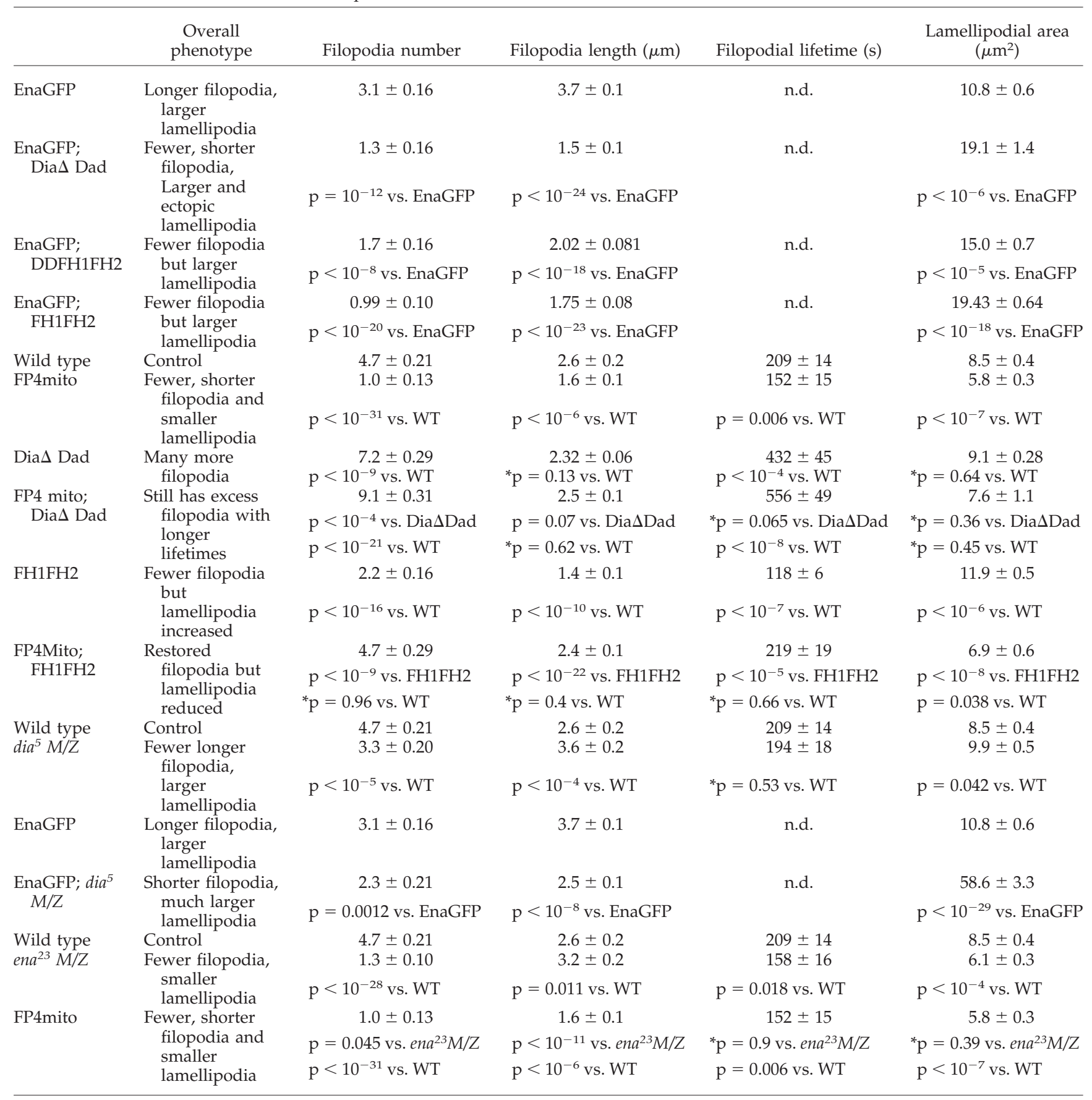

Values are \pm SEM. n.d. $=$ not done.

* Not statistically significant at $p>0.05$.

enous Dia, using $d i a^{5}$ maternal and zygotic mutant embryos $\left(\right.$ dia $\left.^{5} \mathrm{M} / \mathrm{Z}\right)$. One cannot completely eliminate Dia because this disrupts oogenesis (Homem and Peifer, 2008), and severely reduced levels compromise cellularization and subsequent cytokinesis (Afshar et al., 2000). To circumvent this, we used ${d i a^{5}}^{5}$, a temperature-sensitive allele that produces substantially reduced levels of wildtype protein (Homem and Peifer, 2008). To analyze embryos at dorsal closure, we used a temperature-shift strat- egy (Homem and Peifer, 2008). dia ${ }^{5} \mathrm{M} / \mathrm{Z}$ embryos were grown at $18^{\circ} \mathrm{C}$ (the permissive temperature) and then shifted to $25^{\circ} \mathrm{C}$ and analyzed. Many, though not all, early defects accompanying early loss of Dia are overcome by this temperature shift (Homem and Peifer, 2008). It is important to remember when interpreting the experiments below that these embryos retain significant Dia expression and function and thus represent the effects of reducing but not eliminating Dia. 

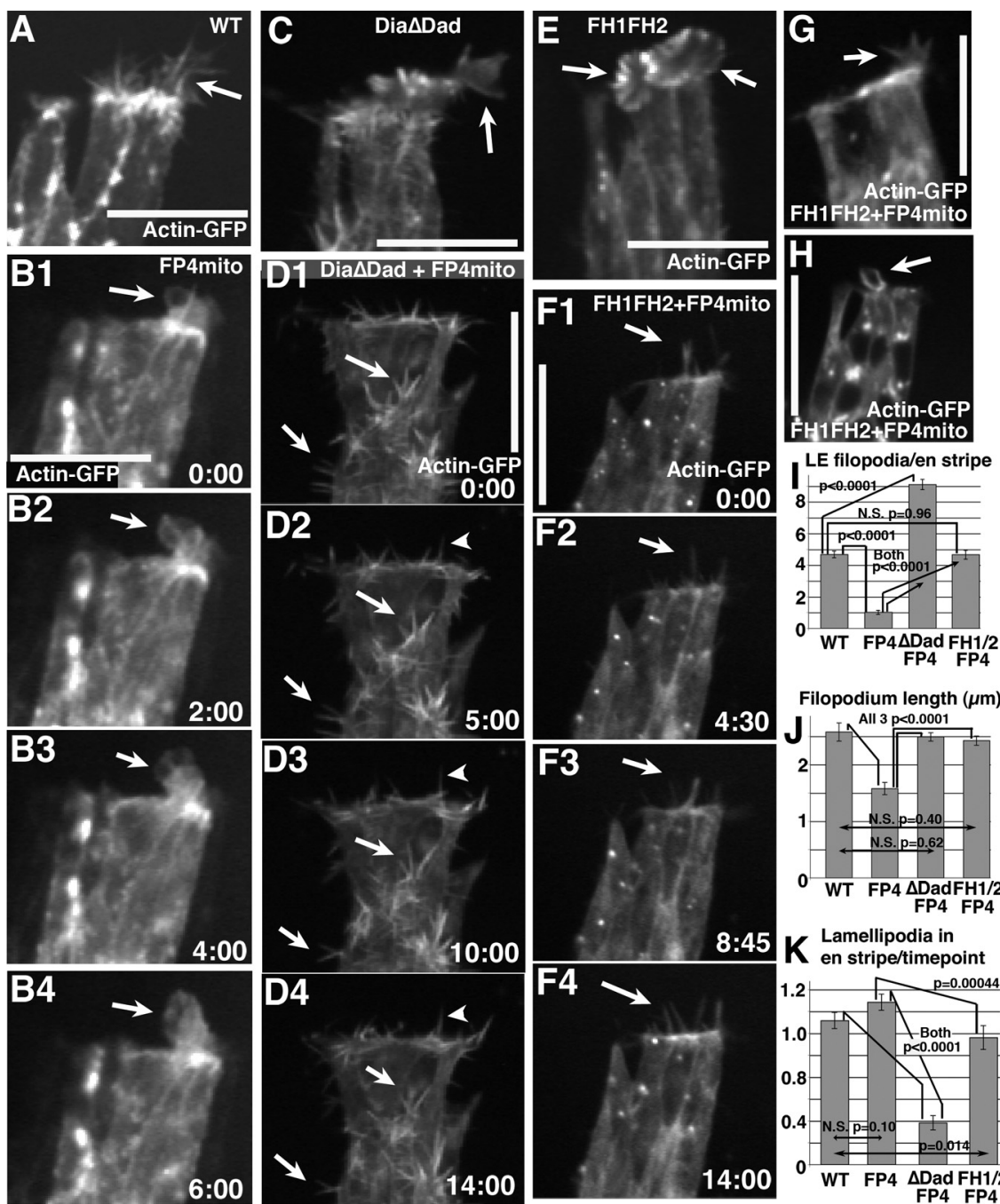

Figure 6. Sequestering Ena while activating Dia reduces lamellipodia but does not eliminate filopodia. (A-H) Movie stills, embryos, stages 14-15, expressing GFP-Actin alone (A) or with FP4mito to sequester Ena (B), Dia $\Delta$ Dad alone (C), Dia $\Delta$ Dad plus FP4Mito (D), FH1FH2 (E), or FH1FH2 plus FP4Mito (F-H), using en-Gal4. Time is shown in minutes:seconds. Scale bars, $10 \mu \mathrm{m}$. (I-K) Quantitation of number of LE filopodia, filopodial length, and number of LE lamellipodia per time point of indicated genotypes. Error bars, SEM.
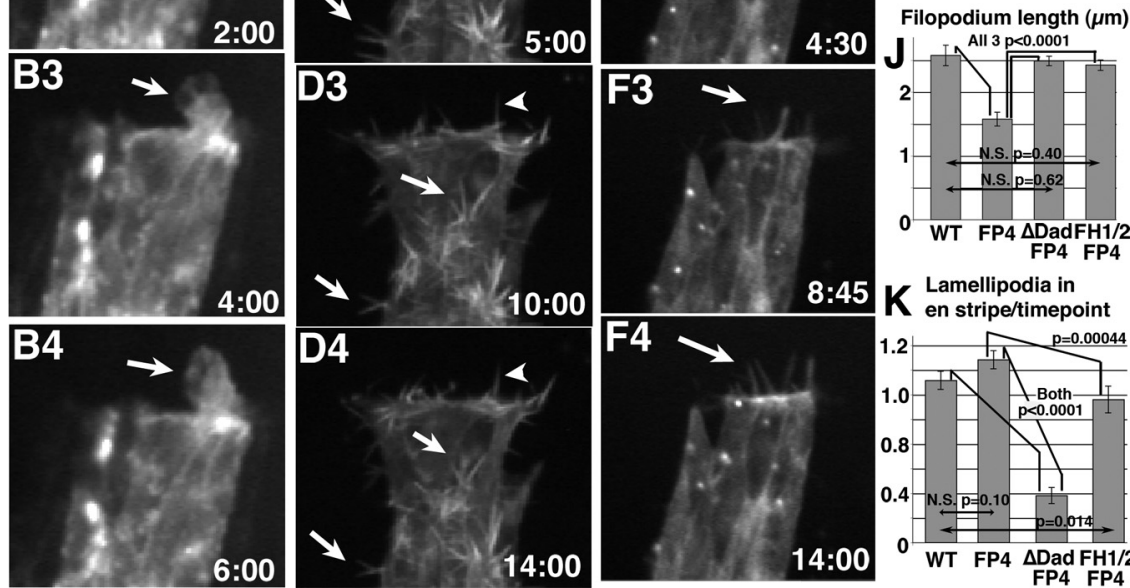

This allowed us to obtain embryos undergoing dorsal closure with reduced Dia levels. Ena localization in dia $^{5} \mathrm{M} / \mathrm{Z}$ embryos was not dramatically altered. Ena still accumulated at LE dots, even in multinucleate cells (Figure 4I; multinucleate cells were rescued by zygotic wild-type Dia; Figure $4 J)$. The subset of $d i a^{5} \mathrm{M} / \mathrm{Z}$ mutants that go far enough to initiate dorsal closure close more slowly than wild type and have defects in epidermal sheet alignment (Figure 7A; data not shown; bright actin structures are forming dorsal hairs). To analyze protrusions in embryos with reduced Dia function, we filmed $\mathrm{dia}^{5} \mathrm{M} / \mathrm{Z}$ embryos expressing Actin-GFP. dia ${ }^{5} \mathrm{M} / \mathrm{Z}$ mutants still form both filopodia and lamellipodia that look surprisingly wild type (Figure 7, B and C; Movie 12), likely due in part to the remaining Dia activity. However, filopodial number was decreased (Figure 7D; Table 3), consistent with a role for Dia in filopodial initiation. Surprisingly, length of the remaining filopodia was increased (Figure $7, \mathrm{C}$ and $\mathrm{E}$; Table 3). In $d i a^{5} \mathrm{M} / \mathrm{Z}$ embryos, the ratio of Dia to Ena should be shifted in favor of Ena; interestingly, lamellipodial area was increased (Figure 7F; Table 3), once again consistent with Ena promoting lamellipodia.

We next explored how further increasing Ena levels affected these protrusions, by overexpressing GFP-Ena in dia ${ }^{5} \mathrm{M} / \mathrm{Z}$ mutants. Our temperature-shift strategy means that Dia activity likely decreases with time (Homem and Peifer, 2008). Although early in dorsal closure GFP-Ena still

localized to filopodial tips in relatively normal protrusions (Figure 7, G vs. H), as epidermal sheets neared one another, distinctive structures were observed: very large lamellipodia with GFP-Ena at the LE (Figure 7I, arrows; lamellipodial area is quantitated in Figure 7L and Table 3). These lamellipodia were very persistent (Figure 7J; Movie 13) and underwent less convergence to form filopodia (Figure 7K; Table 3). These data are also consistent with the idea that when Ena activity exceeds that of Dia, lamellipodia are favored.

\section{Strong Reduction of Ena Reduces Lamellipodia without Eliminating Filopodia}

As a final way of manipulating Ena and Dia levels, we reduced endogenous Ena levels by making embryos maternally and zygotically mutant for the strong but not null allele $e n a^{23}\left(e n a^{23} \mathrm{M} / \mathrm{Z}\right.$; this allele encodes a truncated protein lacking the C-terminal oligimerization domain). These embryos have defects in dorsal closure, with closure substantially slowed and cell matching of the epithelial sheets impaired (Gates et al., 2007). To analyze protrusive behavior of LE cells, we expressed GFP-actin using en-GAL4 in ena ${ }^{23} \mathrm{M} / \mathrm{Z}$ mutants (Figure 8, A-E) and compared them to wild type (Figure $8 \mathrm{~F}$ ) and to embryos expressing FP4mito (Figure 8G), which sequesters Ena. Overall protrusive behavior was substantially reduced in $e n a^{23} \mathrm{M} / \mathrm{Z}$ mutants (Fig- 

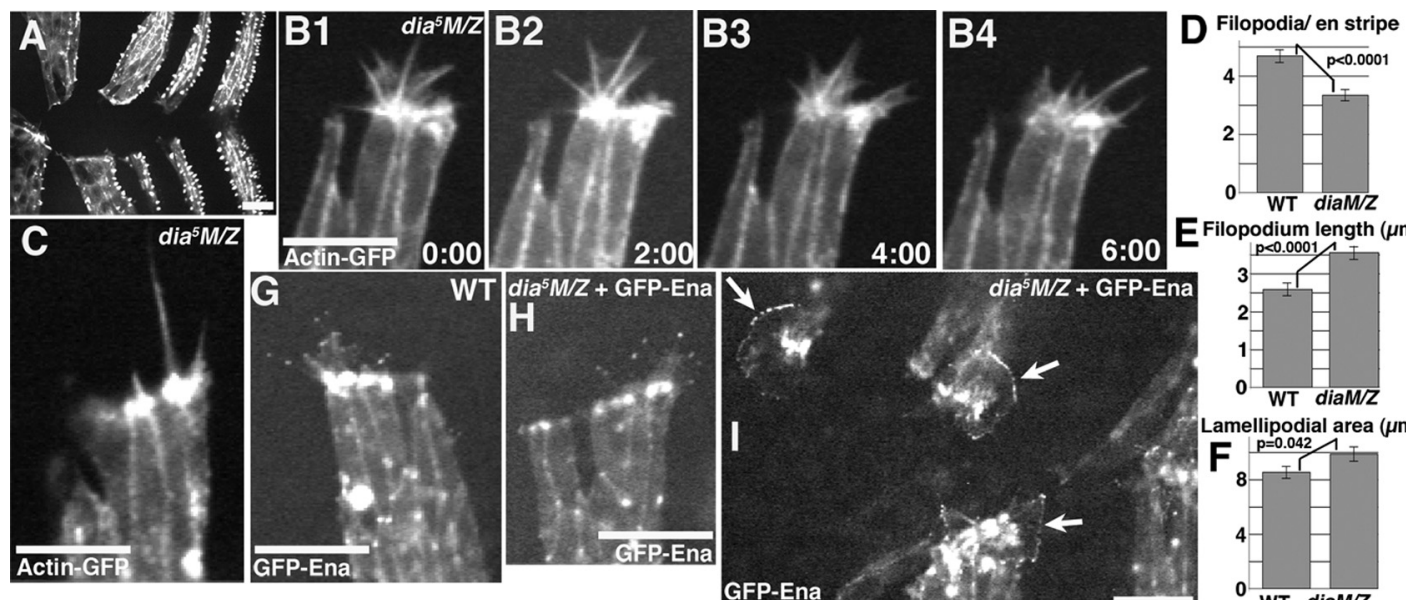

EFilopodium length $(\mu \mathrm{m})$
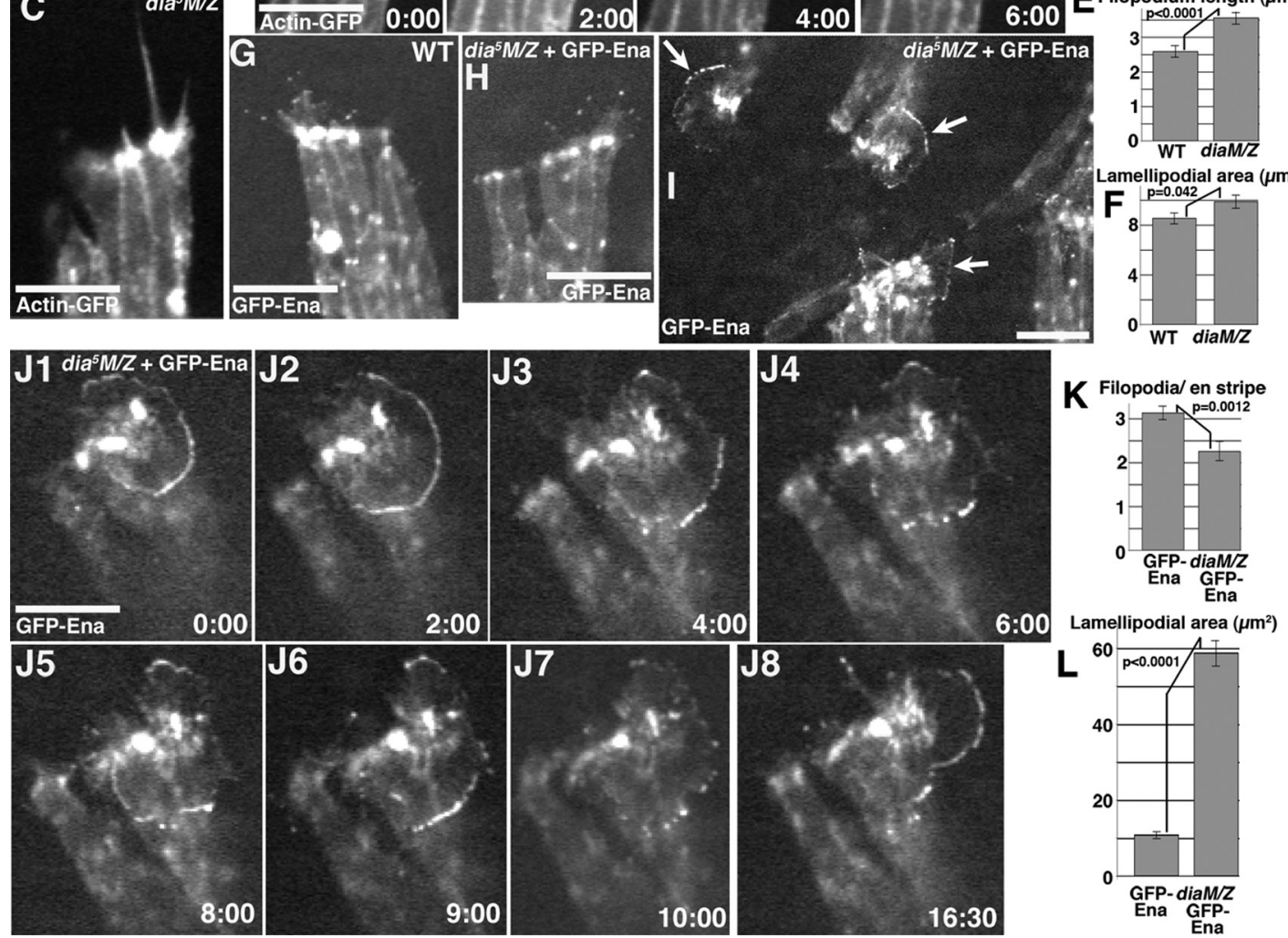

Figure 7. Reducing Dia levels and overexpressing Ena favors lamellipodia. Movie stills, embryos, stages 14-15. Constructs expressed with en-GAL4. (A-C) $\mathrm{dia}^{5} \mathrm{M} / \mathrm{Z}$ mutants expressing actin-GFP. Time is shown in minutes:seconds. (A) Segments fail to align properly during dorsal closure. (B and C) $\mathrm{dia}^{5} \mathrm{M} / \mathrm{Z}$ mutants can make relatively normal protrusions early. (D-F) Quantitation of number of LE filopodia, filopodial length, and lamellipodial area of indicated genotypes. Error bars, SEM. (G) Wild type expressing GFP-Ena. (H-J) dia ${ }^{5}$ M/Z expressing GFP-Ena. (H) Early protrusions can be relatively normal. (I and J) As closure proceeds, large, persistent lamellipodia become common. Scale bars, $10 \mu \mathrm{m}$. (K and L) Quantitation of number of LE filopodia and lamellipodial area of indicated genotypes. Error bars, SEM.

ure 8, A and B; Movie 14). This resulted in part from a large decrease in average lamellipodial area (quantitated in Figure 8J; Table 3), similar to what we observed for FP4mito (Figure 8J; Table 3). Both ena ${ }^{23} \mathrm{M} / \mathrm{Z}$ (Figure 8C, arrowhead) and FP4mito embryos (Figure 8G, arrowhead) also had striking decreases in filopodial number, resulting in "bald lamellipodia" (quantitated in Figure $8 \mathrm{H}$, Table 3 ). There was one striking difference between $e n a^{23} \mathrm{M} / \mathrm{Z}$ mutants and FP4mitoexpressing embryos: in $e n a^{23} \mathrm{M} / \mathrm{Z}$ mutants the remaining filopodia were not reduced in length (Figure 8, D and E, arrows; quantitated in Figure 8I; Table 3), whereas filopodia length was decreased in FP4mito-expressing embryos (Figure 8I; Table 3). Together, these data provide further support for the hypothesis that Ena promotes lamellipodia. They also suggest Ena is important for filopodial number, but may not be essential for filopodial extension. We explore the difference between $e n a^{23} \mathrm{M} / \mathrm{Z}$ and FP4mito further below.

\section{Ena Can Colocalize and Coimmunoprecipitate with Dia and Can Recruit It to Ectopic Locations}

The ability of active Dia to induce Ena relocalization from LE dots to lamellipodia and the ability of Dia and Ena to work together or in parallel to influence protrusive behavior raised the possibility of a direct or indirect interaction between them. Dia and Ena both colocalize to LE dots (Figure 4A). Activated Dia and Ena also localize to filopodial tips in LE cells. These filopodia are not well preserved after fixation of embryos so we further explored filopodial localization in cultured S2 cells. Ena normally localizes to the LE in spreading S2 cells (Supplemental Figure S6A). Dia $\Delta$ Dad can induce long filopodia in these cells (Supplemental Figure 6, B and C), and Ena and GFP-Dia $\Delta$ Dad can colocalize at the tips of these filopodia (Supplemental Figure 6, B and C). We also cotransfected GFP-Dia $\Delta$ Dad and Ena-mCherry and found via live cell imaging that they can colocalize in puncta along filopodia (Supplemental Figure S6D, 1 and 2).

We next explored if Ena is important for Dia's correct localization. We sequestered Ena at mitochondria using FP4mito. In cells expressing FP4mito, we saw a striking and surprising change in Dia localization. Dia is reduced in LE dots (Figure 9A, double arrow), and relocalizes to mitochondria (Figure 9A, arrows, and B); actin is not corecruited there (Figure 9B, inset). To confirm that Dia can be recruited to ectopic locations by Ena, we examined Dia localization in 

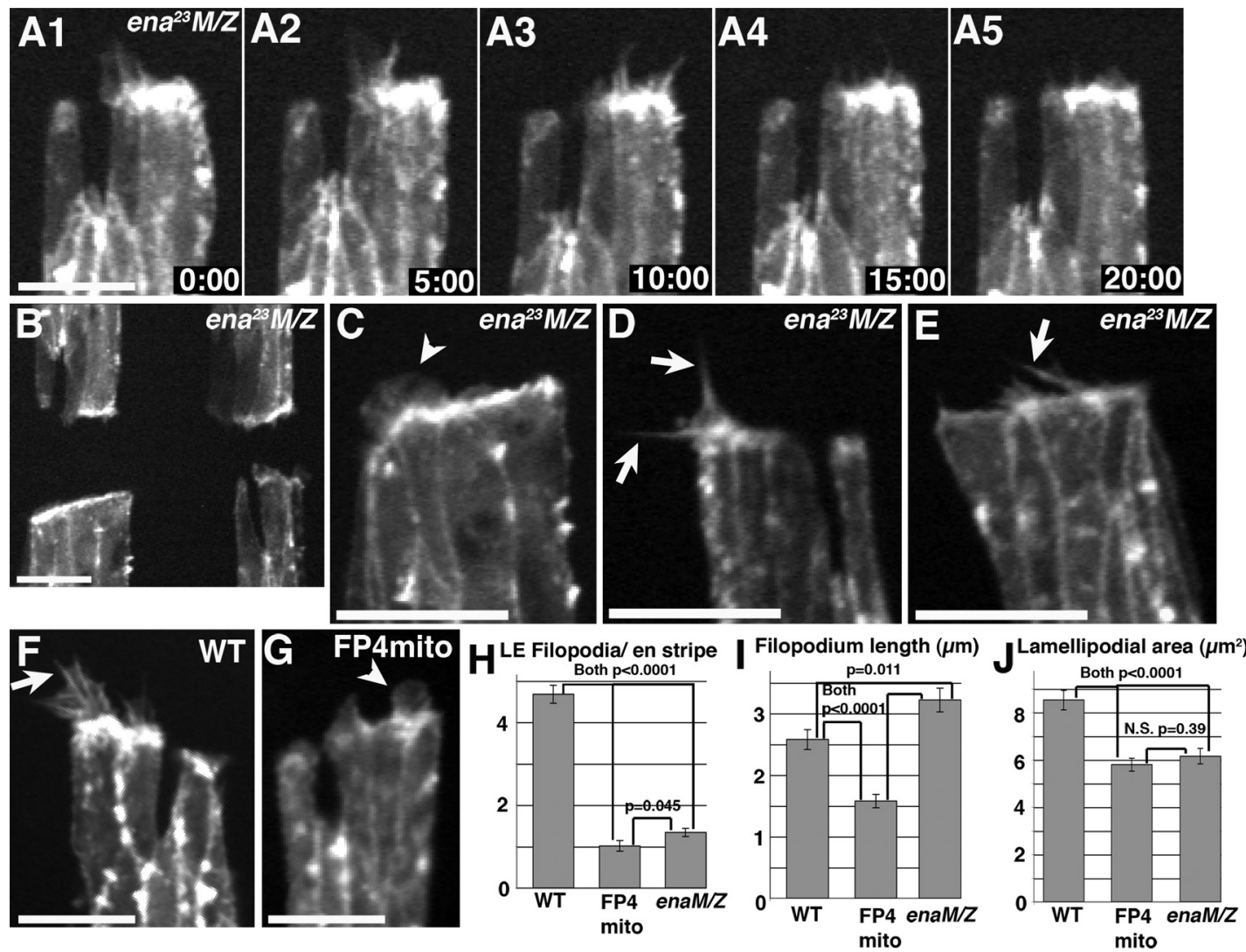

Figure 8. Ena mutants have reduced lamellipodia but still form long filopodia. (A-G) Movie stills, embryos, stages 14-15. Actin-GFP expressed with en-GAL4. Time is shown in minutes:seconds. (A-E) ena ${ }^{23} \mathrm{M} / \mathrm{Z}$ mutant embryos. (F) Wild type. (G) FP4mito. Scale bars, $10 \mu \mathrm{m}$. $(\mathrm{H}-\mathrm{J})$ Quantitation of number of LE filopodia, filopodia length, and lamellipodial area of indicated genotypes. Error bars, SEM.

flies expressing GFP-Ena. GFP-Ena forms cytoplasmic aggregates in expressing cells (Gates et al., 2007). Endogenous Dia also is recruited to these sites (Figure 9C, arrows). These results suggest Ena and Dia may interact and reveal that effects of FP4mito on cell protrusions may reflect not only Ena sequestration but also consequences of Dia mislocalization. This may help explain phenotypic differences between FP4mito and enaM/Z mutants, because, at least in cultured S2 cells, Dia $\Delta$ Dad can still induce filopodia after ena RNAi and can still localize to filopodial tips (Supplemental Figure S6E).

Dia proteins can coimmunoprecipitate (coIP) with VASP in mammals (Grosse et al., 2003) and Dictyostelium (Schirenbeck et al., 2006). We thus tested if fly Ena coIPs with Dia. We IPed Ena from embryos ubiquitously expressing GFP-Ena (using e22c-GAL4), using Ena antibody. Dia coIPed with Ena from these embryos (Figure 9D). We also carried out the reciprocal experiment, IPing Dia from similar embryos with Dia antibody. GFP-Ena coIPed with Dia from these embryos (Figure 9E). Thus Ena can form a direct or indirect complex with Dia.

\section{DISCUSSION}

The actin cytoskeleton underlies many cell behaviors. One critical question is how different actin regulators are coordinated to generate distinct three-dimensional actin lattices needed for different cellular events. We addressed this by exploring how the formin Dia and the Ena/VASP protein Ena work together during cell protrusion, increasing and decreasing activity of each in a single cell type to allow direct comparisons.

\section{The GBD and DD Domains in Dia Play Roles in Intracellular Localization and Function}

Dia is a mosaic of protein domains. The profilin-binding FH1 and actin-binding FH2 domains mediate nucleation and processive anticapping. The FH3 domain is a complex structure including Arm repeats/DID and a DD (Rose et al., 2005). In the standard view, intramolecular interactions between the GBD/DID and Dad keep Dia inactive. Consistent with this, each of our mutants lacking the Dad (Dia $\Delta$ Dad, FH3FH1FH2, DDFH1FH2, and FH1FH2) substantially increased cortical actin and disrupted morphogenesis (Supplemental Figures 2 and 3; Homem and Peifer, 2008).

In other formins the FH3 domain also mediates localization (Zigmond, 2004b), and in DRFs it mediates interaction with partners/regulators including Abi1, WAVE, and IQGAP1 (Brandt et al., 2007; Yang et al., 2007; Beli et al., 2008). We used LE cells to explore how different Dia domains regulate its localization and activity. Full-length Dia is largely cytoplasmic, consistent with the idea that most Dia is in an inactive conformation. In contrast, activating Dia by removing the Dad led to strong cortical recruitment. This suggests relief of intramolecular inhibition involves binding to cortical partners. A pool of active Dia acts at AJs (Homem and Peifer, 2008), consistent with this hypothesis.

Our data suggest the LE is also a site of normal Dia activation. Lamellipodia and filopodia are normally restricted to LE cells and confined to their LE. Dia $\Delta$ Dad in- 

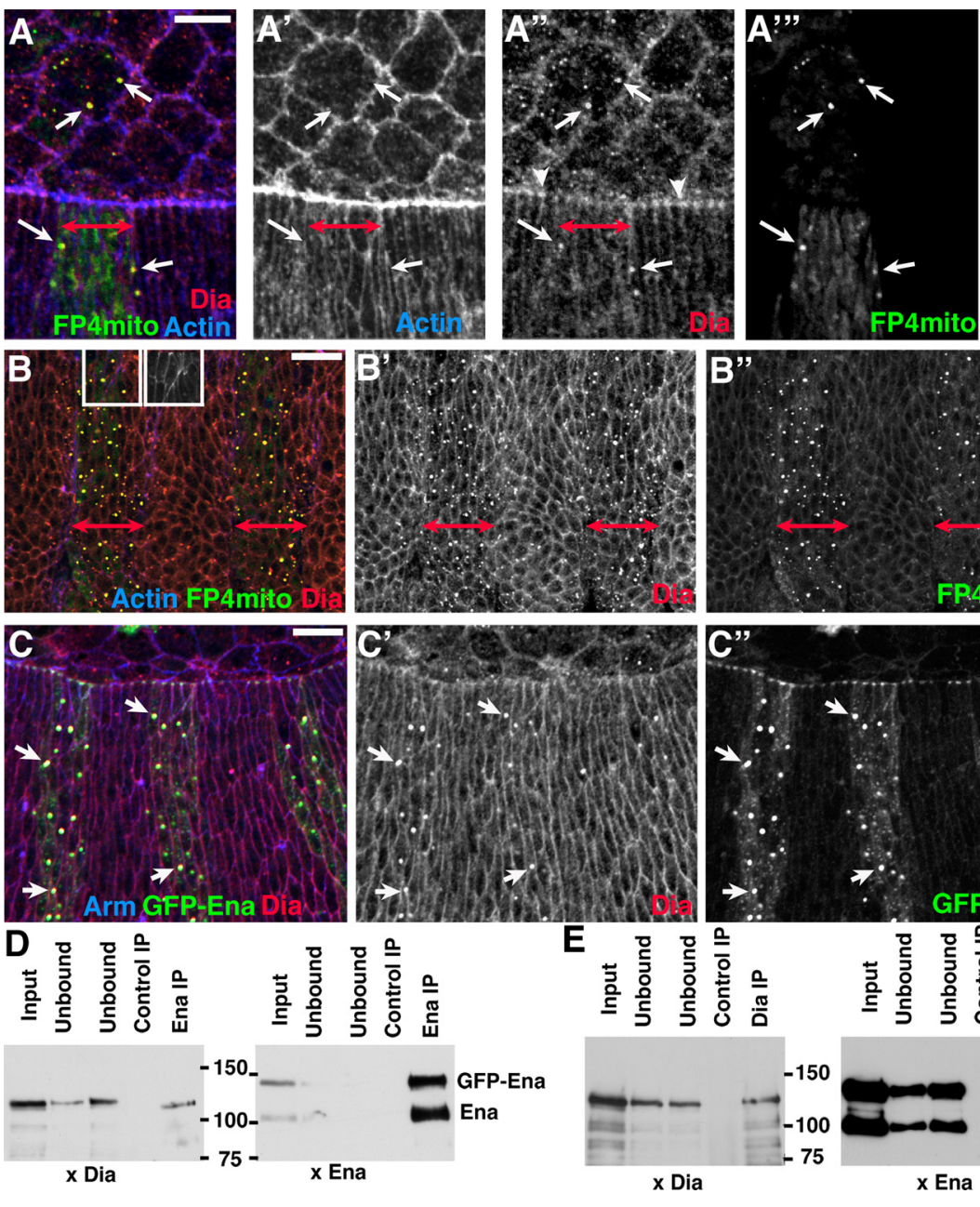

x Ena
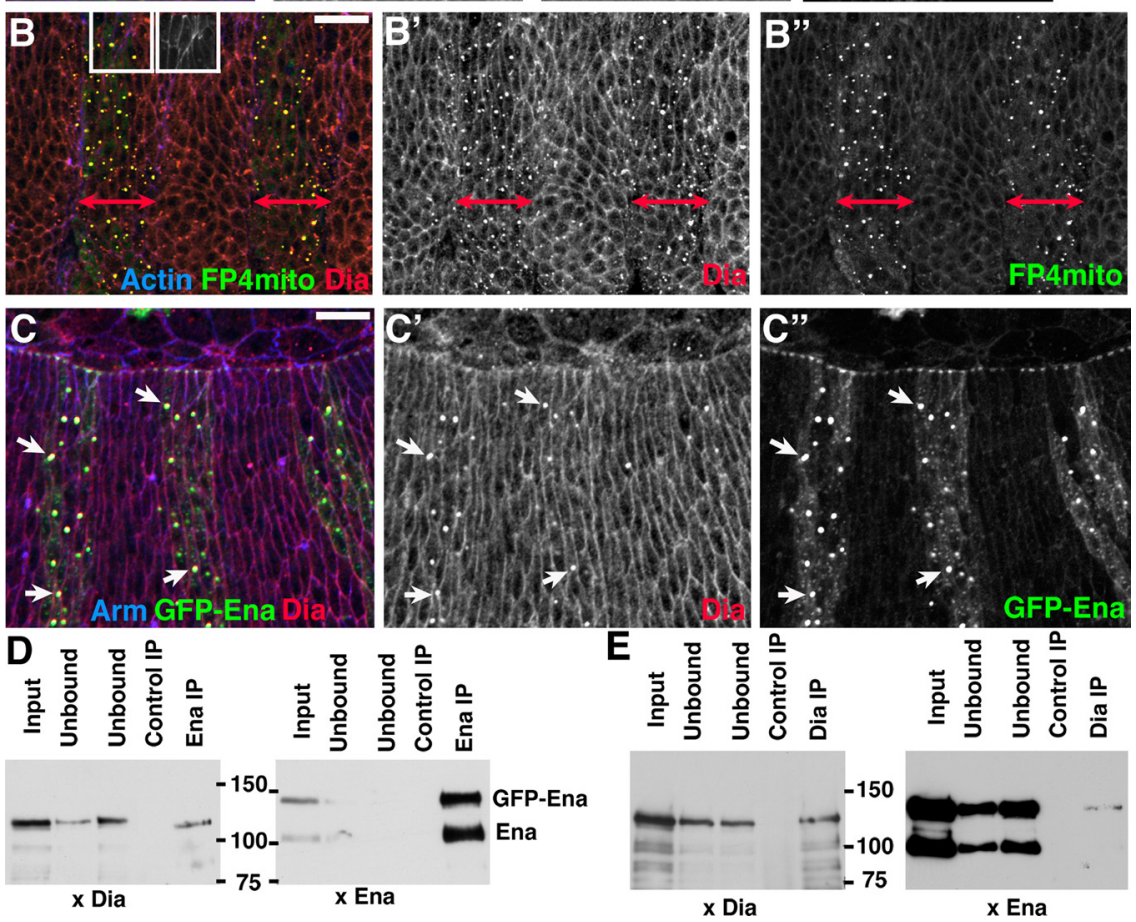

Figure 9. Ena and Dia coIP and Ena can recruit Dia to ectopic locations. (A-C) Embryos, stages 13-15. Antigens and genotypes indicated. (A and B) Embryos expressing GFPFP4Mito under control of en-Gal4. Doubleheaded arrows, FP4Mito expressing stripes. Arrows, Dia colocalizing with GFP-FP4Mito in the cytoplasm. Inset, actin channel in left boxed area. Actin is not recruited by FP4mito. (C) GFP-Ena expressed in paired stripes. Dia is recruited to GFP-Ena cytoplasmic aggregates (arrows). Scale bars, $10 \mu \mathrm{m}$. (D) Embryonic extract from embryos expressing GFP-Ena IPed with a control (anti-myc) antibody or anti-Ena antibody. Dia coIPs with Ena but not with control. (E) Embryonic extract from embryos expressing GFP-Ena IPed with a control (anti-myc) antibody or anti-Dia antibody. GFP-Ena coIPs with Dia but not with control. duced abundant filopodia, as in mammalian cells (Block et al., 2008). Strikingly, these were not confined to the LE, but covered the apical surfaces of LE and more ventral epidermal cells. This suggests Dia is normally activated by a localized signal that is absent or reduced in other epidermal cells, and restricted to the LE. It is likely several inputs mark the LE as special. First, integrated JNK, Dpp, and Wnt signaling specify LE cell fate (Martin and Parkhurst, 2004). Then one must specify the LE of these cells. Signals from amnioserosal cells may help polarize LE cells. Further, the LE is a "free" edge, without cadherin-based cell-cell contact. AJs may inhibit protrusiveness, via Rho family GTPases (Nelson, 2008). Ultimately, it is likely that Rho and perhaps other family members are specifically activated at the LE. Exploring this signaling network, developing tools to measure Rho activity in vivo, and deciphering how these are connected will be important. However, Dia cortical recruitment is not restricted to the LE, and Rho activity is not the sole determinant of cortical Dia recruitment, because cortical Dia was not reduced by dominant-negative Rho.

Although Dia $\Delta$ Dad is largely cortical, FH3FH1FH2, DDFH1FH2, and FH1FH2 are all largely cytoplasmic, suggesting efficient cortical targeting requires the GBD. Thus this domain has functions beyond intramolecular inhibition and its relief by Rho and may bind additional partners. Our data further suggest cortical localization is critical for inducing filopodia, because FH3FH1FH2, DDFH1FH2, and FH1FH2 do not induce ectopic filopodia, although all ele- vate cortical actin and disrupt morphogenesis to the same degree as Dia $\Delta$ Dad. Dia $\Delta$ Dad's ability to induce ectopic filopodia and increase their lifetimes suggests membranetargeted active Dia may be sufficient to initiate and maintain a filopodium. Rho may help recruit Dia to cortical sites and simultaneously activate it, but the maintenance of cortical Dia in cells expressing dominant-negative Rho suggests additional cortical targeting cues exist.

Our data also suggest that active Dia localizes to filopodial tips. Dia $\Delta$ Dad localizes at filopodial tips, like $\mathrm{dDia} 2$ and mDia2 (Schirenbeck et al., 2005b). However, Dia $\Delta$ Dad-induced filopodia are not longer than wild type; perhaps in Dia $\Delta$ Dad, actin monomers are distributed between many more filopodia, limiting growth of each. Consistent with this, DDFH1FH2, which was strongly recruited to filopodial tips, did not induce ectopic filopodia, but filopodia in this background were longer. In contrast, FH1FH2 was less enriched at filopodial tips and did not induce more or longer filopodia. This is consistent with the idea that the DD plays a role in Dia's ability to target tips and elongate filopodia.

We were surprised that removing the DD allowed FH1FH2 to induce large, persistent lamellipodia containing linear actin structures, rather than filopodia. However, mDia2 also regulates linear actin polymerization in lamellipodia (Yang et al., 2007), suggesting a possible explanation. Intriguingly, these ectopic structures were reduced by FP4mito, consistent with the idea that they require Ena for their formation. DDFH1FH2 did not induce these unusual 
lamellipodia, consistent with the idea that the DD and dimerization limits this ectopic activity.

\section{Activating Dia Alters Ena Localization, Perhaps Mobilizing It to Modulate Protrusive Behavior}

Ena/VASP proteins and Dia both regulate actin elongation and filopodia. During dorsal closure, Ena is especially enriched at LE dots, where anterior and posterior edges of LE cells abut the amnioserosa (Gates et al., 2007). This enrichment was puzzling; although Ena/VASP proteins regulate cell adhesion in some mammalian cell types (Vasioukhin et al., 2000; Scott et al., 2006), Ena is not critical for cell adhesion in flies (Gates et al., 2007, 2009). Interestingly, Dia also accumulates at LE dots.

We thus speculated that Ena in LE dots might be inactive but ready for mobilization to induce protrusions at the LE. Consistent with this, all of our activated forms of Dia induce Ena relocalization from LE dots to protrusions. Active Dia may mobilize Ena from LE dots by two mechanisms that are not mutually exclusive: by nucleating new actin filaments and creating barbed ends for which Ena has affinity or by direct or indirect interactions. These possibilities can now be tested. It will also be important to determine if the interaction between Ena and Dia we observed by coIP is direct or indirect and to map the domains required for this interaction.

Sequestering actin regulators at sites along AJs is an attractive mechanism to balance adhesion and motility. Adherent, nonmotile cells must strengthen adhesion and inhibit protrusiveness. Sequestering actin regulators at AJs would prevent them from acting elsewhere, thus inhibiting motility. In contrast, when cells transition from adherent to motile, decreased AJ stability is accompanied by increased protrusiveness and motility. Thus AJs are perfectly placed to coordinate actin rearrangements with cell adhesion. One can now explore mechanisms by which inactive Ena and Dia are retained at LE dots.

Our data also offer a cautionary note to those using FP4mito to sequester Ena. Our data suggest that, at least in Drosophila, FP4mito also sequesters a subset of cellular Dia. Data from both cultured mammalian cells (e.g., Bear et al., 2000) and from Drosophila embryos and ovaries (Gates et al., 2007, 2009) are consistent with the idea that FP4mito mimics Ena/VASP loss of function. However, in Drosophila, we previously found instances where effects of FP4mito were more severe than or qualitatively different from our strongest ena alleles (Gates et al., 2007, 2009). Here we found differences in the effect on protrusive behavior, largely in effects on filopodial length (Table 3). Thus one must remember that one might sequester other actin regulators when using FP4mito.

\section{Roles for Ena and Dia in Tuning the Lamellipodial-Filopodial Balance}

Cells vary widely in the protrusions they produce. Some, like fish keratocytes, are dominated by lamellipodia. Others, like B16 melanoma cells, make lamellipodia with interspersed filopodia, whereas neuronal growth cones and LE cells have lamellipodial veils with prominent filopodia. We explored the roles of Ena and Dia to seek insights into possible mechanistic bases of these differences. Both Ena and Dia increase filopodia when activated/overexpressed alone. We thus expected expressing both together would be additive/synergistic, further increasing filopodial number or length. Instead filopodia were converted into fan-like lamellipodia. This suggests that these actin regulators have different consequences depending on cellular context and the suite of other regulators coexpressed with them.
When we combine our different analyses, several trends emerge. The first is that activated Dia $\Delta$ Dad, with all its N-terminal regulatory domains intact, induces ectopic filopodia and increases filopodial lifetime. It does this in the wild-type background and in cells expressing FP4mito (Tables 2 and 3). This is consistent with the ability of activated Dia to induce and maintain filopodia even when Ena activity is strongly reduced, something that was also observed in mammalian neurons (Dent et al., 2007). However, in wildtype embryos Ena also plays an important role in inducing and maintaining filopodia, as both filopodial number and lifetime are significantly reduced in ena $a^{23} \mathrm{M} / \mathrm{Z}$ mutants (Table 3). Dia's GBD appears to be important for full Dia activity in promoting filopodial, as neither FH3FH1FH2 nor DDFH1FH2 is as effective in increasing filopodial number or lifetime in wild type (Table 2 ).

Second, although expression of Ena in a wild-type background promotes filopodia, in most of our experiments Ena overexpression also promoted lamellipodia, as measured by increased lamellipodial area, whereas sequestering Ena using FP4mito or reducing its activity in ena ${ }^{23} \mathrm{M} / \mathrm{Z}$ mutants both decreased lamellipodial area. This is consistent with the ability of Ena to promote more rapid lamellipodial protrusion in fibroblasts, though in that cell type, these were less persistent (Bear et al., 2002). It also fits with the role of Ena in maintaining a smooth LE in keratocytes (Lacayo et al., 2007).

Although these two properties of Ena and Dia explain many of the effects we see, our data also suggest that relative levels of active Ena and Dia play more complex roles in the type of protrusion formed. For example, simultaneously activating Dia and overexpressing Ena reduced the number of filopodia significantly, although increasing lamellipodial area dramatically. Paradoxically, reducing wild-type Dia (using $\mathrm{dia}^{5} \mathrm{M} / \mathrm{Z}$ mutants) and simultaneously overexpressing Ena also induced very large, persistent lamellipodia, quite distinct from the numerous filopodia produced by overexpressing Ena in wild type. These sorts of paradox may be explained in part if not all structures we call filopodia or call lamellipodia are identical. For example, lamellipodia produced when neither Ena nor Dia is limiting (cells overexpressing Ena and Dia $\Delta$ Dad) may be produced when the entire LE becomes filled with cross-linked linear filaments, forming fan-like lamellipodia like those observed in B16 cells expressing activated Dia (Yang et al., 2007). Activated Dia could increase the number of Dia-generated/ anticapped filaments, and with elevated Ena levels, all might associate with Ena, allowing excess Ena-mediated filament bundling. In contrast, the broad, stable lamellipodia produced by Ena overexpression coupled with Dia reduction may be quite different in structure. With few Dia-generated filaments, most membrane-associated Ena may bind Arp2/3-generated filaments. This would stabilize the LE, but without normal Dia levels, we hypothesize filaments are not effectively gathered into bundles. The result would be a stable, slowly protruding lamellipodium. Likewise, the short, fat filopodia seen after expression of FH1FH2 may be quite distinct from wild-type filopodia, as they appear to largely emerge from fan-like lamellipodia and do not appear to elongate after formation.

How do these experimentally manipulated situations reflect the diverse protrusive behaviors in vivo? Our data suggest that part of this diversity involves balancing Ena and Dia activities. In this speculative view, when Ena activity exceeds Dia's, it may trigger relatively persistent lamellipodial protrusion, as, for example, in fibroblasts. As Dia activity increases, one may transit through lamellipodia with interspersed filopodia, as in B16 melanoma cells, to the 
dynamic, balanced mixture of filopodia and lamellipodia of growth cones or LE cells. Of course, Ena and Dia are part of a much more complex picture. Other key parameters will include Arp2/3 activity (e.g., Le Clainche and Carlier, 2008; Korobova and Svitkina, 2008), CP levels (e.g., Mejillano et al., 2004; Akin and Mullins, 2008), and activity of fascin and other bundling factors, among others. Learning how activities of all these players are integrated is a continuing challenge.

\section{ACKNOWLEDGMENTS}

We thank P. Martin (University of Bristol, Bristol, United Kingdom); the Bloomington Drosophila Stock Center, the Developmental Studies Hybridoma Bank, S. Wasserman (University of California, San Diego, La Jolla, CA), and P. Rorth (Institute of Molecular and Cell Biology, Singapore) for reagents; D. Applewhite, N. Harris, and S. Nowotarski for generously helping with the final experiments; and J. Bear, J. Gates, members of the Peifer and Rogers labs, and the two anonymous reviewers for comments and discussion. C.H. was a student of the Gulbenkian Ph.D. Program in Biomedicine, Portugal. This work was supported by National Institutes of Health Grant RO1 GM47857.

\section{REFERENCES}

Afshar, K., Stuart, B., and Wasserman, S. A. (2000). Functional analysis of the Drosophila diaphanous FH protein in early embryonic development. Development 127, 1887-1897.

Akin, O., and Mullins, R. D. (2008). Capping protein increases the rate of actin-based motility by promoting filament nucleation by the Arp $2 / 3$ complex. Cell 133, 841-851.

Alberts, A. S. (2001). Identification of a carboxyl-terminal diaphanous-related formin homology protein autoregulatory domain. J. Biol. Chem. 276, 28242830 .

Applewhite, D. A., Barzik, M., Kojima, S. I., Svitkina, T. M., Gertler, F. B., and Borisy, G. G. (2007). Ena/VASP proteins have an anti-capping independent function in filopodia formation. Mol. Biol. Cell 18, 2579-2591.

Bachmann, C., Fischer, L., Walter, U., and Reinhard, M. (1999). The EVH2 domain of the vasodilator-stimulated phosphoprotein mediates tetramerization, F-actin binding, and actin bundle formation. J. Biol. Chem. 274, 23549 23557.

Barzik, M., Kotova, T. I., Higgs, H. N., Hazelwood, L., Hanein, D., Gertler, F. B., and Schafer, D. A. (2005). Ena/VASP proteins enhance actin polymerization in the presence of barbed end capping proteins. J. Biol. Chem. 280 $28653-28662$.

Bear, J. E., Loureiro, J. J., Libova, I., Fassler, R., Wehland, J., and Gertler, F. B. (2000). Negative regulation of fibroblast motility by Ena/VASP proteins. Cell 101, 717-728.

Bear, J. E., et al. (2002). Antagonism between Ena/VASP proteins and actin filament capping regulates fibroblast motility. Cell 109, 509-521.

Beli, P., Mascheroni, D., Xu, D., and Innocenti, M. (2008). WAVE and Arp2/3 jointly inhibit filopodium formation by entering into a complex with mDia2. Nat. Cell Biol. 10, 849-857.

Block, J., Stradal, T. E., Hanisch, J., Geffers, R., Kostler, S. A., Urban, E., Small, J. V., Rottner, K., and Faix, J. (2008). Filopodia formation induced by active mDia2/Drf3. J. Microsc. 231, 506-517.

Bloor, J. W., and Kiehart, D. P. (2002). Drosophila RhoA regulates the cytoskeleton and cell-cell adhesion in the developing epidermis. Development 129 3173-3183

Brandt, D. T., Marion, S., Griffiths, G., Watanabe, T., Kaibuchi, K., and Grosse, R. (2007). Dia1 and IQGAP1 interact in cell migration and phagocytic cup formation. J. Cell Biol. 178, 193-200.

Breitsprecher, D., Kiesewetter, A. K., Linkner, J., Urbanke, C., Resch, G. P., Small, J. V., and Faix, J. (2008). Clustering of VASP actively drives processive, WH2 domain-mediated actin filament elongation. EMBO J. 27, 2943-2954.

Carramusa, L., Ballestrem, C., Zilberman, Y., and Bershadsky, A. D. (2007). Mammalian diaphanous-related formin Dia1 controls the organization of E-cadherin-mediated cell-cell junctions. J. Cell Sci. 120, 3870-3882.

Dent, E. W., et al. (2007). Filopodia are required for cortical neurite initiation. Nat. Cell Biol. 9, 1347-1359.

Faix, J., and Grosse, R. (2006). Staying in shape with formins. Dev. Cell 10, 693-706.
Faix, J., and Rottner, K. (2006). The making of filopodia. Curr. Opin. Cell Biol $18,18-25$.

Gates, J., Mahaffey, J. P., Rogers, S. L., Emerson, M., Rogers, E. M., Sottile, S. L., Van Vactor, D., Gertler, F. B., and Peifer, M. (2007). Enabled plays key roles in embryonic epithelial morphogenesis in Drosophila. Development 134, 2027-2039.

Gates, J., Nowotarski, S. H., Yin, H., Mahaffey, J. P., Bridges, T., Herrera, C. Homem, C.C.F., Janody, F., Montell, D. J., and Peifer, M. (2009). Enabled and Capping protein play important roles in shaping cell behavior during Drosophila oogenesis. Dev. Biol. 333, 90-107.

Goode, B. L., and Eck, M. J. (2007). Mechanism and function of formins in the control of actin assembly. Annu. Rev. Biochem. 76, 593-627.

Gorfinkiel, N., and Martinez-Arias, A. (2007). Requirements for adherens junction components in the interaction between epithelial tissues during dorsal closure in Drosophila. J. Cell Sci. 120, 3289-3298.

Grosse, R., Copeland, J. W., Newsome, T. P., Way, M., and Treisman, R (2003). A role for VASP in RhoA-Diaphanous signalling to actin dynamics and SRF activity. EMBO J. 22, 3050-3061.

Homem, C. C., and Peifer, M. (2008). Diaphanous regulates myosin and adherens junctions to control cell contractility and protrusive behavior during morphogenesis. Development 135, 1005-1018.

Huttelmaier, S., Harbeck, B., Steffens, O., Messerschmidt, T., Illenberger, S. and Jockusch, B. M. (1999). Characterization of the actin binding properties of the vasodilator-stimulated phosphoprotein VASP. FEBS Lett. 451, 68-74.

Jacinto, A., Wood, W., Balayo, T., Turmaine, M., Martinez-Arias, A., and Martin, P. (2000). Dynamic actin-based epithelial adhesion and cell matching during Drosophila dorsal closure. Curr. Biol. 10, 1420-1426.

Korobova, F., and Svitkina, T. (2008). Arp2/3 complex is important for filopodia formation, growth cone motility, and neuritogenesis in neuronal cells. Mol. Biol. Cell 19, 1561-1574.

Krause, M., et al. (2004). Lamellipodin, an Ena/VASP ligand, is implicated in the regulation of lamellipodial dynamics. Dev. Cell 7, 571-583.

Lacayo, C. I., Pincus, Z., VanDuijn, M. M., Wilson, C. A., Fletcher, D. A., Gertler, F. B., Mogilner, A., and Theriot, J. A. (2007). Emergence of large-scale cell morphology and movement from local actin filament growth dynamics. PLoS Biol. 5, e233.

Laurent, V., Loisel, T. P., Harbeck, B., Wehman, A., Grobe, L., Jockusch, B. M., Wehland, J., Gertler, F. B., and Carlier, M. F. (1999). Role of proteins of the Ena/VASP family in actin-based motility of Listeria monocytogenes. J. Cell Biol. 144, 1245-1258.

Le Clainche, C., and Carlier, M. F. (2008). Regulation of actin assembly associated with protrusion and adhesion in cell migration. Physiol. Rev. 88 $489-513$.

Lebrand, C., Dent, E. W., Strasser, G. A., Lanier, L. M., Krause, M., Svitkina, T. M., Borisy, G. G., and Gertler, F. B. (2004). Critical role of Ena/VASP proteins for filopodia formation in neurons and in function downstream of netrin-1. Neuron 42, 37-49.

Magie, C. R., Pinto-Santini, D., and Parkhurst, S. M. (2002). Rho1 interacts with p120(ctn) and alpha-catenin, and regulates cadherin- based adherens junction components in Drosophila. Development 129, 3771-3782.

Martin, P., and Parkhurst, S. M. (2004). Parallels between tissue repair and embryo morphogenesis. Development 131, 3021-3034.

Mattila, P. K., and Lappalainen, P. (2008). Filopodia: molecular architecture and cellular functions. Nat. Rev. Mol. Cell Biol. 9, 446-454.

Mejillano, M. R., Kojima, S., Applewhite, D. A., Gertler, F. B., Svitkina, T. M. and Borisy, G. G. (2004). Lamellipodial versus filopodial mode of the actin nanomachinery: pivotal role of the filament barbed end. Cell 118, 363-373.

Nelson, W. J. (2008). Regulation of cell-cell adhesion by the cadherin-catenin complex. Biochem. Soc. Trans. 36, 149-155.

Pellegrin, S., and Mellor, H. (2005). The Rho family GTPase Rif induces filopodia through mDia2. Curr. Biol. 15, 129-133.

Peng, J., Wallar, B. J., Flanders, A., Swiatek, P. J., and Alberts, A. S. (2003) Disruption of the Diaphanous-related formin Drf1 gene encoding mDia1 reveals a role for Drf3 as an effector for Cdc42. Curr. Biol. 13, 534-545.

Pollard, T. D. (2007). Regulation of actin filament assembly by arp2/3 complex and formins. Annu. Rev. Biophys. Biomol. Struct. 36, 451-477.

Pollard, T. D., and Borisy, G. G. (2003). Cellular motility driven by assembly and disassembly of actin filaments. Cell 112, 453-465.

Pruyne, D., Evangelista, M., Yang, C., Bi, E., Zigmond, S., Bretscher, A., and Boone, C. (2002). Role of formins in actin assembly: nucleation and barbedend association. Science 297, 612-615. 
Ridley, A. J. (2006). Rho GTPases and actin dynamics in membrane protrusions and vesicle trafficking. Trends Cell Biol. 16, 522-529.

Rorth, P. (1998). Gal4 in the Drosophila female germline. Mech. Dev. 78, $113-118$.

Rose, R., Weyand, M., Lammers, M., Ishizaki, T., Ahmadian, M. R., and Wittinghofer, A. (2005). Structural and mechanistic insights into the interaction between Rho and mammalian Dia. Nature 435, 513-518.

Ryu, J. R., Echarri, A., Li, R., and Pendergast, A. M. (2009). Regulation of cell-cell adhesion by Abi/Diaphanous complexes. Mol. Cell Biol. 29, 17351748.

Sagot, I., Rodal, A. A., Moseley, J., Goode, B. L., and Pellman, D. (2002). An actin nucleation mechanism mediated by Bni1 and profilin. Nat. Cell Biol. 4 , 626-631.

Samarin, S., Romero, S., Kocks, C., Didry, D., Pantaloni, D., and Carlier, M. F. (2003). How VASP enhances actin-based motility. J. Cell Biol. 163, 131-142.

Schirenbeck, A., Arasada, R., Bretschneider, T., Schleicher, M., and Faix, J. (2005a). Formins and VASPs may co-operate in the formation of filopodia. Biochem. Soc. Trans. 33, 1256-1259.

Schirenbeck, A., Arasada, R., Bretschneider, T., Stradal, T. E., Schleicher, M., and Faix, J. (2006). The bundling activity of vasodilator-stimulated phosphoprotein is required for filopodium formation. Proc. Natl. Acad. Sci. USA 103, 7694-7699.

Schirenbeck, A., Bretschneider, T., Arasada, R., Schleicher, M., and Faix, J. (2005b). The Diaphanous-related formin dDia2 is required for the formation and maintenance of filopodia. Nat. Cell Biol. 7, 619-625.
Scott, J. A., Shewan, A. M., den Elzen, N. R., Loureiro, J. J., Gertler, F. B., and Yap, A. S. (2006). Ena/VASP proteins can regulate distinct modes of actin organization at cadherin-adhesive contacts. Mol. Biol. Cell 17, 1085-1095.

Sheffield, M., Loveless, T., Hardin, J., and Pettitt, J. (2007). C. elegans Enabled exhibits novel interactions with N-WASP, Abl, and cell-cell junctions. Curr. Biol. 17, 1791-1796.

Somogyi, K., and Rorth, P. (2004). Evidence for tension-based regulation of Drosophila MAL and SRF during invasive cell migration. Dev. Cell 7, 85-93.

Steffen, A., Faix, J., Resch, G. P., Linkner, J., Wehland, J., Small, J. V., Rottner, K., and Stradal, T. E. (2006). Filopodia formation in the absence of functional WAVE- and Arp2/3-complexes. Mol. Biol. Cell 17, 2581-2591.

Svitkina, T. M., Bulanova, E. A., Chaga, O. Y., Vignjevic, D. M., Kojima, S. Vasiliev, J. M., and Borisy, G. G. (2003). Mechanism of filopodia initiation by reorganization of a dendritic network. J. Cell Biol. 160, 409-421.

Vasioukhin, V., Bauer, C., Yin, M., and Fuchs, E. (2000). Directed actin polymerization is the driving force for epithelial cell- cell adhesion. Cell 100 209-219.

Yang, C., Czech, L., Gerboth, S., Kojima, S., Scita, G., and Svitkina, T. (2007). Novel roles of formin mDia2 in lamellipodia and filopodia formation in motile cells. PLoS Biol. 5, e317.

Zigmond, S. H. (2004a). Beginning and ending an actin filament: control at the barbed end. Curr. Top. Dev. Biol. 63, 145-188.

Zigmond, S. H. (2004b). Formin-induced nucleation of actin filaments. Curr. Opin. Cell Biol. 16, 99-105.

Zigmond, S. H., Evangelista, M., Boone, C., Yang, C., Dar, A. C., Sicheri, F., Forkey, J., and Pring, M. (2003). Formin leaky cap allows elongation in the presence of tight capping proteins. Curr. Biol. 13, 1820-1823. 